\title{
LDGM-Based Multiple Description Coding for Finite Alphabet Sources
}

\author{
Ying Zhang, Sorina Dumitrescu, Member, IEEE, Jun Chen, Member, IEEE, and Zhibin Sun
}

\begin{abstract}
This work presents an LDGM-based practical successive coding scheme for the multiple description (MD) problem for finite alphabet sources. The scheme, which targets the ZhangBerger $(\mathrm{ZB})$ rate-distortion region, is shown to be asymptotically optimal with joint typicality encoding, while as a practical encoding solution a message passing algorithm is adopted.

We further discuss in more detail the application of the coding scheme in three cases of the MD problem with the Hamming distortion measure: 1) no excess sum-rate for binary sources, 2) successive refinement, and 3) no excess marginal rate for the uniform binary source. In the no excess sum-rate case some progress is made in the characterization of fundamental limits by deriving the analytical expression of the distortion region for general binary sources, and of the auxiliary variables needed to achieve its boundary. The exact expression of the Zhang-Berger upper bound to the central distortion is also provided for the case of no excess marginal rate for the uniform binary source.

The proposed LDGM-based coding scheme is tested in practice for all three aforementioned cases. The experimental results show very good performance, demonstrating its ability to approach the theoretical rate-distortion limits or the available upper bounds.
\end{abstract}

Index Terms-Lossy source coding, low-density generator matrix code, message-passing algorithm, multiple description coding.

\section{INTRODUCTION}

$\mathbf{I}$ $\mathrm{N}$ the multiple description (MD) problem, a source sequence is compressed into two descriptions, which are constructed in such a way that an adequate reconstruction of the source is possible based on each description while the two descriptions together can lead to better reconstruction quality. Information theoretical results on this problem can be found in [1]-[7].

Research on practical MD coding has intensified in recent years due to its applications in modern communications systems [8]-[12]. However, the design of practical schemes able to approach the known fundamental limits for finite alphabet sources has received little attention. For such sources, practical codes based on low-density generator matrices (LDGM), combined with various message passing algorithms, have been successful in approaching the rate-distortion bound for single

Paper approved by Z. Xiong, the Editor for Distributed Coding and Processing of the IEEE Communications Society. Manuscript received December 1, 2011; revised May 4 and June 24, 2012.

This work was presented in part at the 47th Annual Allerton Conference on Communication, Control, and Computing, Monticello, IL, Oct. 2009, the IEEE Information Theory Workshop, Taormina, Italy, Oct. 2009, and the International Conference on Wireless Communications and Signal Processing, Nanjing, China, Nov. 2009.

The authors are with the Department of Electrical and Computer Engineering, McMaster University, Hamilton, ON, L8S 4K1, Canada (e-mail: yingzhang@grads.ece.mcmaster.ca, \{sorina, junchen\}@mail.ece.mcmaster.ca, sunz4@mcmaster.ca).

Digital Object Identifier 10.1109/TCOMM.2012.082812.110817 description source coding. In [13], [14], LDGM codes are used to encode a uniform binary source, while in [17] a multilevel LDGM coding scheme for finite alphabet sources with general distortion measure is proposed.

Motivated by the success of LDGM codes for the single description problem we attempt to apply them to the MD case. However, for finite alphabet sources with general distortion measure the characterization of the MD rate-distortion region is not known. Therefore, we turn our attention to the available inner bounds of the MD region. The first general inner bound, referred to as the EGC region, was provided by El Gamal and Cover in [3]. This was shown to be tight for the no excess sum-rate case by Ahlswede [4]. Later Zhang and Berger [5] proposed a different inner bound, termed the ZB region, and showed that it contains points not included in the EGC region. Recently, Wang et al. [7] proved that the ZB region includes the EGC region and established the $\mathrm{ZB}$ region as the best inner bound known to date. Motivated by the latter result we design an LDGM-based coding scheme for this region. The scheme consists of three sequential encoders and is applicable to corner points of a certain rate region derived from the ZB region, while any other rate pair can be obtained through timesharing of two such corner points. We prove that the coding scheme is asymptotically optimal when the joint typicality rule is employed at each encoder, whereas as a practical solution for the encoding problem we propose a message passing algorithm.

The proposed coding scheme needs knowledge of the auxiliary variables involved in the $\mathrm{ZB}$ region. In order to determine the optimal auxiliary variables achieving rate-distortion points on the boundary of the $\mathrm{ZB}$ region, numerical techniques can be used to solve the underlying non-convex optimization problem. On the other hand, availability of analytical solutions would spare this step and thus simplify the design of the coding system. Therefore, next we turn our attention to several cases of the MD problem with Hamming distortion measure where such optimal auxiliary variables can be computed analytically.

The first case is the no excess sum-rate case for binary sources. In this case the characterization of the MD ratedistortion region is available, as the EGC region, however, the computation of the rate-distortion limits is not simple and analytical expressions are only partly available for the uniform binary source. Interestingly, it turns out that the distortion region and the auxiliary variables achieving its boundary can be computed analytically for general binary sources, and we derive their exact expression.

Further, we consider the case of no excess marginal rate 
for the uniform binary source. For this case Zhang and Berger proposed in [6] a method to compute an upper bound for the central distortion given the values of the side distortions. We improve Zhang and Berger's result by providing the analytical expression of this upper bound and of the achieving auxiliary variables.

Another special case of the MD problem, where the fundamental limits are known, is the problem of successive refinement (SR) for finite alphabet sources [18]-[20]. In this case the second description is of no interest alone, but only in conjunction with the first description. We show how the proposed LDGM-based coding scheme can be adapted to the SR problem.

Finally, we test the proposed coding scheme in each of the aforementioned cases of the MD problem. Our simulations show very good performance demonstrating thus its ability to approach in practice the theoretical rate-distortion limits or the available upper bounds.

We mention that LDGM-based successive coding schemes for the MD problem were also presented in [21], [22]. The scheme in [22] is specifically designed for a Gaussian source with squared-error distortion measure, leveraging on particularities of the MD problem conferred by the specific source and distortion measure, and cannot be extended to the case of finite alphabet sources with general distortion. On the other hand, the MD scheme of [21] is tailored for the EGC region, which can be strictly smaller than the $\mathrm{ZB}$ region, targeted in our work. For instance, Zhang and Berger's upper bound on the central distortion, in the no excess marginal rate case for the uniform binary source, cannot be achieved using the MD code of [21]. Additionally, beside the differences in the coding scheme, another contribution of the current work versus [21], [22] consists in the analytical results emphasized in the previous paragraphs.

The paper is organized as follows. Section II reviews the $\mathrm{MD}$ problem and the $\mathrm{ZB}$ region. The following section presents the proposed sequential coding scheme for the $\mathrm{ZB}$ region. Section IV addresses the no excess sum-rate case for binary sources and derives the analytical expression of the distortion region and boundary achieving auxiliary variables. Section $\mathrm{V}$ discusses the upper bound on the central distortion in the no excess marginal rate case for the uniform binary source. Section VI reviews the SR rate-distortion region and clarifies the application of the proposed coding scheme in this case. Finally, Section VII presents our simulation results and Section VIII concludes the paper.

\section{THE MD PROBLEM}

The formal definition of the MD problem is given as follows. Consider a sequence $X^{n}=(X(1), X(2), \cdots, X(n))$ drawn from an i.i.d. source $X$ with generic distribution $p_{X}$. Let $d: \mathcal{X} \times \hat{\mathcal{X}} \rightarrow[0, \infty)$ be a distortion measure, where $\mathcal{X}$ and $\hat{\mathcal{X}}$ are the source alphabet and the reconstruction alphabet, respectively. Throughout this work, the alphabets $\mathcal{X}$ and $\hat{\mathcal{X}}$ are assumed to be finite. The quintuple $\left(R_{1}, R_{2}, d_{1}, d_{2}, d_{0}\right)$ is said achievable, if for all sufficiently large $n$, there exist encoding functions

$$
f_{t}^{(n)}: \mathcal{X}^{n} \rightarrow\left\{1,2, \cdots,\left\lfloor 2^{n R_{t}}\right\rfloor\right\}, \quad t=1,2,
$$

and decoding functions

$$
\begin{aligned}
& g_{t}^{(n)}:\left\{1,2, \cdots,\left\lfloor 2^{n R_{t}}\right\rfloor\right\} \rightarrow \hat{\mathcal{X}}^{n}, \quad t=1,2, \\
& g_{0}^{(n)}:\left\{1,2, \cdots,\left\lfloor 2^{n R_{1}}\right\rfloor\right\} \times\left\{1,2, \cdots,\left\lfloor 2^{n R_{2}}\right\rfloor\right\} \rightarrow \hat{\mathcal{X}}^{n},
\end{aligned}
$$

such that

$$
\mathbb{E}\left[\frac{1}{n} \sum_{l=1}^{n} d\left(X(l), \hat{X}_{t}(l)\right)\right] \leq d_{t}, \quad t=0,1,2,
$$

where

$$
\begin{aligned}
& \hat{X}_{t}^{n}=g_{t}^{(n)}\left(f_{t}^{(n)}\left(X^{n}\right)\right), \quad t=1,2, \\
& \hat{X}_{0}^{n}=g_{0}^{(n)}\left(f_{1}^{(n)}\left(X^{n}\right), f_{2}^{(n)}\left(X^{n}\right)\right) .
\end{aligned}
$$

The MD rate-distortion region, denoted by $\mathcal{R} \mathcal{D}$, is the closure of the set of all achievable quintuples $\left(R_{1}, R_{2}, d_{1}, d_{2}, d_{0}\right)$.

Zhang and Berger [5] proposed an inner bound of the MD rate-distortion region termed the $\mathrm{ZB}$ region. The $\mathrm{ZB}$ region, denoted by $\mathcal{R} \mathcal{D}_{\mathrm{ZB}}$, is the set of quintuples $\left(R_{1}, R_{2}, d_{1}, d_{2}, d_{0}\right)$ such that there exist random variables $X_{c}, X_{1}, X_{2}$ jointly distributed with $X$ and functions $\psi_{t}, t=0,1,2$, satisfying

$$
\begin{aligned}
& R_{t} \geq I\left(X ; X_{c}, X_{t}\right), \quad t=1,2, \\
& R_{1}+R_{2} \geq 2 I\left(X ; X_{c}\right)+I\left(X ; X_{1}, X_{2} \mid X_{c}\right) \\
& \quad+I\left(X_{1} ; X_{2} \mid X_{c}\right), \\
& \quad \mathbb{E} d\left(X, \psi_{t}\left(X_{c}, X_{t}\right)\right) \leq d_{t}, \quad t=1,2, \\
& \mathbb{E} d\left(X, \psi_{0}\left(X_{c}, X_{1}, X_{2}\right)\right) \leq d_{0} .
\end{aligned}
$$

The question whether $\mathcal{R} \mathcal{D}_{\mathrm{ZB}}$ is tight is an open problem. However, $\mathcal{R} \mathcal{D}_{\mathrm{ZB}}$ is the best inner bound known so far for $\mathcal{R} \mathcal{D}$. Therefore, in the next section we propose a practical coding scheme based on LDGM codes for the $\mathrm{ZB}$ region.

\section{AN LDGM-BASED CODING SCHEME FOR THE}

\section{Corner Rate Points Associated to The ZB Region}

\section{A. Sequential Random Coding Scheme}

Given the auxiliary random variables $X_{c}, X_{1}, X_{2}$ jointly distributed with $X$, the distortion triple $\left(d_{1}, d_{2}, d_{0}\right)$ obeying (3) and (4) can be achieved by the set of rate pairs $\left(R_{1}, R_{2}\right)$ satisfying relations (1) and (2). Let us denote by $\mathcal{R}\left(p_{X_{c} X_{1} X_{2} \mid X}\right)$ this rate region, and by $\mathcal{F}\left(p_{X_{c} X_{1} X_{2} \mid X}\right)$ its dominant face, which is defined as the set of rate pairs $\left(R_{1}, R_{2}\right) \in \mathcal{R}\left(p_{X_{c} X_{1} X_{2} \mid X}\right)$ for which (2) holds with equality. Without loss of generality, we shall focus on $\mathcal{F}\left(p_{X_{c} X_{1} X_{2} \mid X}\right)$ since every point in $\mathcal{R}\left(p_{X_{c} X_{1} X_{2} \mid X}\right)$ is dominated, in a componentwise sense, by some point $\left(R_{1}, R_{2}\right) \in \mathcal{F}\left(p_{X_{c} X_{1} X_{2} \mid X}\right)$. Moreover, the two corner points $E_{1}$ and $E_{2}$ of $\mathcal{R}\left(p_{X_{c} X_{1} X_{2} \mid X}\right)$ (i.e., the end points of $\left.\mathcal{F}\left(p_{X_{c} X_{1} X_{2} \mid X}\right)\right)$ are of particular importance. Specifically, one can achieve an arbitrary point on the dominant face by timesharing $E_{1}$ and $E_{2}$. Precisely, the coordinates of $E_{1}$ are

$$
\begin{aligned}
& R_{1}\left(E_{1}\right)=I\left(X ; X_{c}\right)+I\left(X ; X_{1} \mid X_{c}\right), \\
& R_{2}\left(E_{1}\right)=I\left(X ; X_{c}\right)+I\left(X, X_{1} ; X_{2} \mid X_{c}\right) .
\end{aligned}
$$

The coordinates of $E_{2}$ are obtained by swapping indices 1 and 2 in the above relations.

The successive encoding scheme directly suggested by (5) needs conditional codebooks, which complicate the implementation. To avoid this we use a variable substitution as follows. 


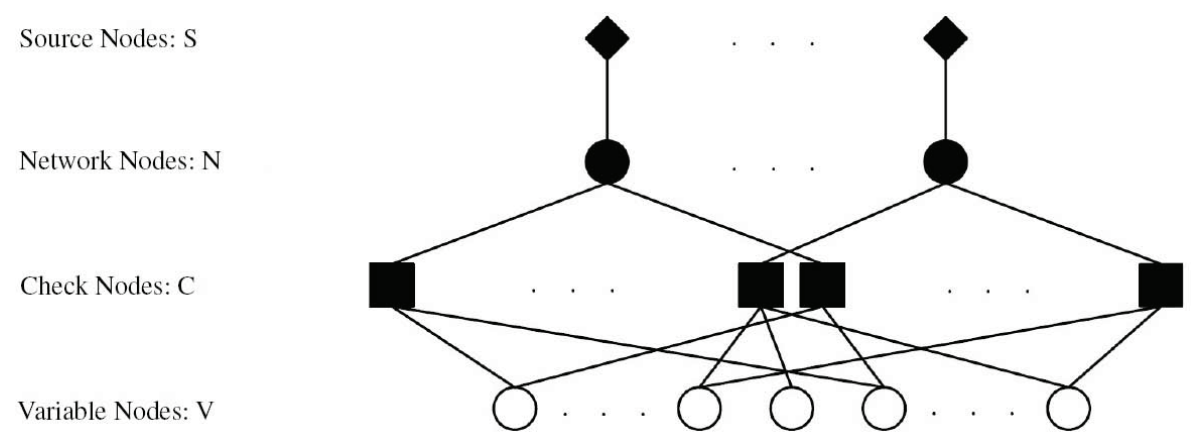

Fig. 1. Factor graph associated to the multilevel LDGM code.

According to [7, Lemma 1], there exist random variables $U_{t}^{1}$, $t=1,2$, taking values in the finite sets $\mathcal{U}_{t}$, respectively, with $\left|\mathcal{U}_{t}\right| \leq\left|\mathcal{X}_{c}\right|\left(\left|\mathcal{X}_{t}\right|-1\right)+1, t=1,2$, and functions $\pi_{t}: \mathcal{X}_{c} \times \mathcal{U}_{t} \rightarrow \mathcal{X}_{t},, t=1,2$, such that

C1) $U_{t}$ is independent of $X_{c}$;

C2) $X_{t}=\pi_{t}\left(X_{c}, U_{t}\right)$;

C3) $\left(X, X_{2}\right)-\left(X_{c}, X_{1}\right)-U_{1}$ and $\left(X, X_{1}, U_{1}\right)-\left(X_{c}, X_{2}\right)-U_{2}$ form Markov chains.

The above relations imply that

$$
\begin{gathered}
I\left(X, X_{c} ; U_{1}\right)=I\left(X_{c} ; U_{1}\right)+I\left(X ; U_{1} \mid X_{c}\right)=I\left(X ; X_{1} \mid X_{c}\right) \\
I\left(X, X_{c}, X_{1} ; U_{2}\right)=I\left(X_{c} ; U_{2}\right)+I\left(X, X_{1} ; U_{2} \mid X_{c}\right) \\
=I\left(X, X_{1} ; X_{2} \mid X_{c}\right) .
\end{gathered}
$$

Hence, (5) can be written equivalently as

$$
\begin{aligned}
& R_{1}\left(E_{1}\right)=I\left(X ; X_{c}\right)+I\left(X, X_{c} ; U_{1}\right), \\
& R_{2}\left(E_{1}\right)=I\left(X ; X_{c}\right)+I\left(X, X_{c}, X_{1} ; U_{2}\right) .
\end{aligned}
$$

Next we present a sequential coding scheme approaching the corner point $E_{1}$. This system consists of three encoders, labeled 0,1 and 2 .

Codebook generation. Encoder 0 randomly generates a codebook $\mathcal{C}_{0}=\left\{x_{c, i}^{n}\right\}_{i=1}^{n\left(I\left(X ; X_{c}\right)+\epsilon\right)}$, according to the distribution $\prod_{l=1}^{n} p_{X_{c}}(\cdot)$. Encoder 1 randomly generates a codebook

$\mathcal{C}_{1}=\left\{u_{1, j}^{n}\right\}_{j=1}^{n\left(I\left(X, X_{c} ; U_{1}\right)+\epsilon\right)}$, according to the distribution $\prod_{l=1}^{n} p_{U_{1}}(\cdot)$. Encoder 2 randomly generates a codebook $\mathcal{C}_{2}=\left\{u_{2, k}^{n}\right\}_{k=1}^{n\left(I\left(X, X_{c}, X_{1} ; U_{2}\right)+\epsilon\right)}$, according to the distribution $\prod_{l=1}^{n} p_{U_{2}}(\cdot)$.

Encoding. Given the source sequence $x^{n}$, encoder 0 selects a codeword $x_{c, i}^{n}$ which is jointly strongly typical with $x^{n}$. Encoder 1 finds an index $j$ such that $u_{1, j}^{n}$ is jointly strongly typical with $x^{n}$ and $x_{c, i}^{n}$. Finally, encoder 2 chooses an index $k$ such that $u_{2, k}^{n}$ is jointly strongly typical with $x^{n}, x_{c, i}^{n}$, and $x_{1, i, j}^{n}$, where $x_{1, i, j}^{n}=\left(\pi_{1}\left(x_{c, i}(l), u_{1, j}(l)\right)\right)_{l=1}^{n}$. The pairs $(i, j)$ and $(i, k)$ form descriptions 1 and 2 , respectively.

Decoding. Decoder 1 receives index pair $(i, j)$ and takes $g_{1}^{(n)}(i, j)=\left(\psi_{1}\left(x_{1, i, j}^{n}(l)\right)\right)_{l=1}^{n}$ as the reconstruction. Decoder 2 receives index pair $(i, k)$ and reconstructs the source as $g_{2}^{(n)}(i, k)=\left(\psi_{2}\left(x_{2, i, k}^{n}(l)\right)\right)_{l=1}^{n}$, where $x_{2, i, k}^{n}(l)=\pi_{2}\left(x_{c, i}(l), u_{2, k}(l)\right)$. The central decoder obtains the reconstruction as $g_{0}^{(n)}(i, j, i, k)=$ $\left(\psi_{0}\left(x_{c, i}(l), x_{1, i, j}^{n}(l), x_{2, i, k}^{n}(l)\right)\right)_{l=1}^{n}$.

${ }^{1}$ The construction of such variables is shown in the proof of Lemma 1 in
Following the above sequential coding system we propose an LDGM-based coding scheme for the corner rate points $E_{1}$ and $E_{2}$, which uses a multilevel LDGM code to generate the codebook at each encoding stage. Further, we show that when the joint typicality rule is used at each encoder the proposed scheme achieves optimality asymptotically. Finally, we present the message passing algorithm used as a practical heuristic solution for the encoding problem at each stage.

\section{B. Asymptotically Optimal LDGM-based Code}

A multilevel LDGM code of rate $R=\frac{m}{n}$ is specified by a low density generator matrix $G$ of dimension $m \times n \omega$ over $\mathbb{F}_{2}$ and a mapping $\phi: \mathbb{F}_{2}^{\omega} \rightarrow \mathcal{Z}$, for some positive integer $\omega$ and finite set $\mathcal{Z}$. Define now the mapping $\Phi: \mathbb{F}_{2}^{n \omega} \rightarrow \mathcal{Z}^{n}$ such that for any $c^{n \omega} \in \mathbb{F}_{2}^{n \omega}$, the $l$-th component of $\Phi\left(c^{n \omega}\right)$ is $\phi(c(l), c(n+l), \cdots, c(n(\omega-1)+l))$, for $1 \leq l \leq n$. Then the multilevel LDGM code specified by $G$ and $\phi$ is defined as the set

$$
\mathcal{C}(G, \phi) \triangleq\left\{\Phi\left(v^{m} G\right) \mid v^{m} \in \mathbb{F}_{2}^{m}\right\},
$$

where $v^{m} G$ denotes the product between the row vector $v^{m}$ and the matrix $G$.

Consider now the joint probability distribution $p_{Y, \phi(\tilde{Y})}$, where $Y$ takes values in some finite alphabet $\mathcal{Y}$, while $\tilde{Y}$ takes values in $\mathbb{F}_{2}^{\omega}$. Then we define the joint typicality encoder associated to $\mathcal{C}(G, \phi)$ and $p_{Y, \phi(\tilde{Y})}$ as the mapping $\mathcal{E}_{G, \phi, p_{Y, \phi(\tilde{Y})}}: \mathcal{Y}^{n} \rightarrow \mathbb{F}_{2}^{m}$, where

$$
\begin{aligned}
& \mathcal{E}_{G, \phi, p_{Y, \phi(\tilde{Y})}}\left(y^{n}\right) \triangleq \\
& \quad \operatorname{argmin}_{v^{m} \in \mathbb{F}_{2}^{m}}\left\{\alpha:\left(y^{n}, \Phi\left(v^{m} G\right)\right) \in \mathcal{T}_{p_{Y, \phi(\tilde{Y})}^{\alpha, n}}^{\alpha,}\right\} .
\end{aligned}
$$

Note that for every $\alpha>0$ and every distribution $p_{V_{1}, V_{2}}$, $\mathcal{T}_{p_{V_{1}, V_{2}}}^{\alpha, n}$ denotes the set of pairs of sequences $\left(v_{1}^{n}, v_{2}^{n}\right)$ which are $\alpha$-strongly jointly typical according to $p_{V_{1}, V_{2}}{ }^{2}$. Likewise, $\mathcal{T}_{p_{V_{1}}}^{\alpha, n}$ denotes the set of sequences $v_{1}^{n}$ which are $\alpha$-strongly typical according to $p_{V_{1}}$.

Further, as in [17] we consider an LDGM code ensemble specified by a random generator matrix $\mathbf{G}$ with elements selected independently from $\mathbb{F}_{2}$ using a Bernoulli distribution with parameter $p_{n}$, where $p_{n} \rightarrow 0$ and $n p_{n} \rightarrow \infty$ as $n \rightarrow \infty$. Finally, following [17], [15] and [16], we obtain the following result, which is crucial for our development and whose proof is given in Appendix A.

\footnotetext{
${ }^{2}$ The definition of strongly typical sequences is as in [24].
} 


$$
\begin{aligned}
M_{S_{l} \rightarrow N_{l}}(z)= & \exp (-\gamma(y(l), z)), \text { for all } z \in \mathcal{Z}, 1 \leq l \leq n, \\
M_{N_{l} \rightarrow C_{l+s n}}(b)= & \sum_{z \in \mathcal{Z}} M_{S_{l} \rightarrow N_{l}}(z) \sum_{\substack{b^{\omega} \in\{0,1\} \omega \\
b(s+1)=b \\
\phi(b \omega)=z}} \prod_{\substack{j=0 \\
j \neq s}}^{\omega-1} M_{C_{l+j n} \rightarrow N_{l}}(b(j+1)), \\
M_{C_{l+s n} \rightarrow N_{l}}(b)= & \frac{1}{2}+\frac{(-1)^{b}}{2} \prod_{\substack{k \in \mathcal{B}_{v}(l+s n)\\
}}\left(M_{V_{k} \rightarrow C_{l+s n}}(0)-M_{V_{k} \rightarrow C_{l+s n}}(1)\right), \\
& \text { for all } b \in\{0,1\}, 1 \leq l \leq n, 0 \leq s \leq \omega-1, \quad \prod_{i \in \mathcal{B}_{v}(l+s n) \backslash\{k\}}\left(M_{V_{i} \rightarrow C_{l+s n}}(0)-M_{V_{i} \rightarrow C_{l+s n}}(1)\right), \\
M_{C_{l+s n} \rightarrow V_{k}}(b)= & \frac{1}{2}+\frac{(-1)^{b}}{2}\left(M_{N_{l} \rightarrow C_{l+s n}}(0)-M_{N_{l} \rightarrow C_{l+s n}}(1)\right) \quad \prod_{M_{V_{k} \rightarrow C_{l+s n}}(b)=} \\
& \quad \text { for all } b \in\{0,1\}, k \in \mathcal{A}_{c}(k) \backslash\{l+s n\} \\
M_{C_{q} \rightarrow V_{k}}(b), &
\end{aligned}
$$

Fig. 2. Message passing equations. The message passed by node $A$ to node $B$ is denoted by $M_{A \rightarrow B}$. It is a vector with two components for $A \neq S_{l}$, and with $|\mathcal{Z}|$ components for $A=S_{l}$. After applying these equations, the components of each message are normalized to sum up to 1 . $\mathcal{A}_{c}(k)$ denotes the set of indices $q$ such that $C_{q}$ is adjacent to node $V_{k}$, and $\mathcal{B}_{v}(q)$ denotes the set of of indices $k$ such that $V_{k}$ is adjacent to $C_{q}$. The values of the parameters $\gamma(y, z)$ used in our simulations are specified in Section VII.

Lemma 1. Consider the LDGM code ensemble as described above for some $R, \omega, \phi, Y$, and $\tilde{Y}$ such that $\tilde{Y}$ is uniformly distributed over $\mathbb{F}_{2}^{\omega}$ and $R \geq I(Y ; \tilde{Y})+\epsilon_{0}$, for some arbitrary $\epsilon_{0}>0$. Furthermore, assume that the random vector $Y^{n}$ representing the input at the encoder satisfies the equality

$$
\lim _{n \rightarrow \infty} \mathbb{P}\left(Y^{n} \in \mathcal{T}_{p_{Y}}^{\delta, n}\right)=1,
$$

for any $\delta>0$. Then, for any $\delta>0$ one has

$$
\lim _{n \rightarrow \infty} \mathbb{P}\left(\left(Y^{n}, \Phi\left(\mathcal{E}_{\mathbf{G}, \phi, p_{Y, \phi(\tilde{Y})}}\left(Y^{n}\right) \mathbf{G}\right)\right) \in \mathcal{T}_{p_{Y, \phi(\tilde{Y})}^{\delta, n}}^{\delta, ~}\right)=1 .
$$

In order to proceed with the specifics of the proposed LDGM-based code for each encoding stage, we need first the following result which was proved in [17] as a part of the proof of Theorem 1 .

Lemma 2 ( [17]). Consider arbitrary jointly distributed random variables $Y$ and $Z$ over the finite alphabets $\mathcal{Y}$ and $\mathcal{Z}$, respectively. Then, for any positive integer $\omega$ there exist a random variable $\tilde{Y}_{\omega}$ uniformly distributed over $\mathbb{F}_{2}^{\omega}$ and a mapping $\phi_{\omega}: \mathbb{F}_{2}^{\omega} \rightarrow \mathcal{Z}$ such that

$$
\lim _{\omega \rightarrow \infty} p_{Y, \phi_{\omega}\left(\tilde{Y}_{\omega}\right)}(y, z)=p_{Y, Z}(y, z)
$$

for every $(y, z) \in \mathcal{Y} \times \mathcal{Z}$.

In virtue of the above result for each triple of positive integers $\omega_{0}, \omega_{1}, \omega_{2}$ there exist random variables $\tilde{X}_{0, \omega_{0}}, \tilde{X}_{1, \omega_{0}, \omega_{1}}$ and $\tilde{X}_{2, \omega_{0}, \omega_{1}, \omega_{2}}$ uniformly distributed over $\mathbb{F}_{2}^{\omega_{0}}, \mathbb{F}_{2}^{\omega_{1}}$, and $\mathbb{F}_{2}^{\omega_{2}}$ respectively, and mappings $\phi_{0}: \mathbb{F}_{2}^{\omega_{0}} \rightarrow \mathcal{X}_{c}, \phi_{1}: \mathbb{F}_{2}^{\omega_{1}} \rightarrow \mathcal{U}_{1}$, and $\phi_{2}: \mathbb{F}_{2}^{\omega_{2}} \rightarrow \mathcal{U}_{2}$ such that

$$
\begin{aligned}
& \lim _{\omega_{0} \rightarrow \infty} p_{X, \phi_{0}\left(\tilde{X}_{0, \omega_{0}}\right), U_{1}, U_{2}}\left(x, x_{c}, u_{1}, u_{2}\right) \\
& =p_{X, X_{c}, U_{1}, U_{2}}\left(x, x_{c}, u_{1}, u_{2}\right)
\end{aligned}
$$

$$
\begin{aligned}
& \lim _{\omega_{1} \rightarrow \infty} p_{X, \phi_{0}\left(\tilde{X}_{0, \omega_{0}}\right), \phi_{1}\left(\tilde{X}_{1, \omega_{0}, \omega_{1}}\right), U_{2}}\left(x, x_{c}, u_{1}, u_{2}\right) \\
& =p_{X, \phi_{0}}\left(\tilde{X}_{0, \omega_{0}}\right), U_{1}, U_{2} \\
& \left(x, x_{c}, u_{1}, u_{2}\right)
\end{aligned}
$$

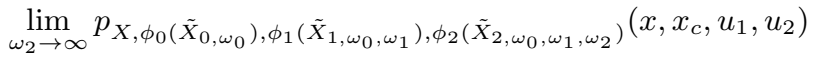

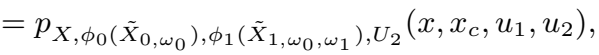

for every $\left(x, x_{c}, u_{1}, u_{2}\right) \in \mathcal{X} \times \mathcal{X}_{c} \times \mathcal{U}_{1} \times \mathcal{U}_{2}$.

Let $\omega_{j}$, respectively $\phi_{j}$, denote the value of $\omega$, respectively the mapping $\phi$ used at encoder $j$, for $j=0,1,2$. The encoding mapping at each stage is defined as in (6) where

a) $Y=X$ and $\tilde{Y}=\tilde{X}_{0, \omega_{0}}$ at encoder 0 ;

b) $Y=\left(X, \phi_{0}\left(\tilde{X}_{0, \omega_{0}}\right)\right)$ and $\tilde{Y}=\tilde{X}_{1, \omega_{0}, \omega_{1}}$ at encoder 1 ;

c) $Y=\left(X, \phi_{0}\left(\tilde{X}_{0, \omega_{0}}\right), \pi_{1}\left(\phi_{0}\left(\tilde{X}_{0, \omega_{0}}\right), \phi_{1}\left(\tilde{X}_{1, \omega_{0}, \omega_{1}}\right)\right)\right)$ and $\tilde{Y}=\tilde{X}_{2, \omega_{0}, \omega_{1}, \omega_{2}}$ at encoder 2 .

The rates at the three encoders are as in the previous subsection, i.e.

$$
\begin{aligned}
& R_{(0)}=I\left(X ; X_{c}\right)+\epsilon \text { at encoder } 0 ; \\
& R_{(1)}=I\left(X, X_{c} ; U_{1}\right)+\epsilon \text { at encoder } 1 ; \\
& R_{(2)}=I\left(X, X_{c}, X_{1} ; U_{2}\right)+\epsilon \text { at encoder } 2 .
\end{aligned}
$$

Lemmas 1 and 2 immediately imply the asymptotical optimality of the proposed LDGM-based coding scheme, which is stated next.

Theorem 1. The sequential LDGM-based coding scheme described in this subsection ensures that the target distortion values $d_{1}, d_{2}$ and $d_{0}$ for the side and central descriptions, respectively, can be approached arbitrarily closely as $n, \omega_{0}, \omega_{1}, \omega_{2} \rightarrow \infty$.

Proof of Theorem 1. Notice that in view of relations (9-11) and of the continuity of the information mapping $I(\cdot, \cdot)$, there exists some positive $\epsilon_{0}(\epsilon)$ such that the condition $R_{(j)} \geq$ $I(Y ; \tilde{Y})+\epsilon_{0}$ is satisfied at every encoder $j=0,1,2$, for sufficiently large $\omega_{j}$ and the corresponding pair of random variables $(Y ; \tilde{Y})$. Since condition (7) is satisfied for encoder 0 it follows that Lemma 1 can be applied, thus relation (8) holds as well. This further implies that condition (7) is also valid at encoder 1, consequently (8) also holds for encoder 1, which validates (7) for encoder 2 as well. Finally, by Lemma 
1, (8) also holds for encoder 2. Combining these observations with (9-11) the conclusion of Theorem 1 follows.

Unfortunately, solving the encoder problem (6) is impractical, therefore we use as a heuristic solution the belief propagation with decimation on the factor graph associated to the LDGM code (Fig. 1). This algorithm is similar to the message passing algorithm described in [14], [17], [21], [22]. However, a notable distinction versus the aforementioned prior work stems from the difference in the objective of the encoder. Namely, while in the single description setting the objective is to minimize the distortion between the codeword and the input sequence, in our case the objective is to find a codeword which is strongly jointly typical with the input sequence. To account for this difference we will use more parameters in the computation of the messages passed by the source nodes as seen in Fig. 2. For an attempt to justify this strategy we refer the reader to [23]. The justification in [23] is based on heuristically modeling problem (6) as the problem of minimizing a weighted sum of frequencies of pairs of letters $(y, z) \in \mathcal{Y} \times \mathcal{Z}$, in $\left(y^{n}, \Phi\left(v^{m} G\right)\right)$, for some appropriate weights. Then the parameters used in the definition of the messages passed by the source nodes in Fig. 2 correspond to these weights.

The factor graph is composed of $n$ source nodes $\left\{S_{1}, \cdots, S_{n}\right\}, m$ variable nodes $\left\{V_{1}, \cdots, V_{m}\right\}, n \omega$ check nodes $\left\{C_{1}, \cdots, C_{n \omega}\right\}$, and $n$ network nodes $\left\{N_{1}, \cdots, N_{n}\right\}$. Every variable node $V_{k}$ is associated to information bit $v(k)$ and is connected by an edge to every check node $C_{h}$ such that $G(k, h)=1$. Each network node $N_{l}$ is connected by an edge to check nodes $C_{l}, C_{l+n}, \cdots, C_{l+(\omega-1) n}$, and to source node $S_{l}$. Source node $S_{l}$ is associated to the $l$-th symbol $y(l)$ in the input sequence, and $N_{l}$ is associated to the $l$-th symbol $z(l)$ of the output sequence (i.e., codeword). Finally, the equations to compute the messages are presented in Fig. 2 and the algorithm description in Fig. 3.

\section{Distortion Region for the No Excess Sum-RAte} CASE FOR BINARY SOURCES With HAMMING Distortion

The term "no excess sum-rate" refers to the case when $R_{1}+$ $R_{2}=R\left(d_{0}\right)$, where $R(\cdot)$ denotes the rate-distortion function. As shown by Ahlswede in [4], the following relation holds

$$
\begin{aligned}
& \left\{\left(R_{1}, R_{2}, d_{1}, d_{2}, d_{0}\right) \in \mathcal{R D}: R_{1}+R_{2}=R\left(d_{0}\right)\right\}= \\
& \left\{\left(R_{1}, R_{2}, d_{1}, d_{2}, d_{0}\right) \in \mathcal{Q}: R_{1}+R_{2}=R\left(d_{0}\right)\right\},
\end{aligned}
$$

where $\mathcal{Q}$ denotes the convex closure of the set of quintuples $\left(R_{1}, R_{2}, d_{1}, d_{2}, d_{0}\right)$ for which there exist auxiliary random variables $X_{t}, t=0,1,2$, jointly distributed with $X$ such that

$$
\begin{aligned}
& I\left(X_{1} ; X_{2}\right)=0, \\
& R_{t} \geq I\left(X ; X_{t}\right), \quad t=1,2, \\
& R_{1}+R_{2} \geq I\left(X ; X_{0}, X_{1}, X_{2}\right), \\
& d_{t} \geq \mathbb{E}\left[d\left(X, X_{t}\right)\right], \quad t=0,1,2 .
\end{aligned}
$$

Our next result represents some progress towards an explicit characterization of $\mathcal{R D}$ in the no excess sum-rate case for general binary sources with Hamming distortion measure (i.e., $d(x, \hat{x})=d_{H}(x, \hat{x})=0$ if $x=\hat{x}$, and 1 otherwise). Let $\mathcal{X}=\hat{\mathcal{X}}=\{0,1\}$ and $p_{X}(0)=\delta$, where $0<\delta \leq 1 / 2$. Let $\mathcal{D}$
1: $\max _{\text {iter }} \leftarrow 100$

2: $\mathcal{A}_{c}(k) \leftarrow\{q \mid G(k, q)=1\}$, for all $k=1, \cdots, m$

3: $\mathcal{B}_{v}(q) \leftarrow\{k \mid G(k, q)=1\}$, for all $q=1, \cdots, n \omega$

4: $\mathcal{N} \mathcal{V} \leftarrow\{1, \cdots, m\}$

5: for $l=1$ to $n$ do

Compute $M_{S_{l} \rightarrow N_{l}}$ as in Fig. 2

for $s=0$ to $\omega-1$ do

$M_{C_{l+s n} \rightarrow N_{l}} \leftarrow\left(\frac{1}{2}, \frac{1}{2}\right)$

for $k \in \mathcal{B}_{v}(l+s n)$ do

$M_{C_{l+s n} \rightarrow V_{k}} \leftarrow\left(\frac{1}{2}, \frac{1}{2}\right)$

end for

end for

\section{end for}

while $\mathcal{N} \mathcal{V} \neq \emptyset$ do

for $i=1$ to max $_{i t e r}$ do

Compute $M_{N_{l} \rightarrow C_{l+s n}}, M_{V_{k} \rightarrow C_{l+s n}}$ as in Fig. 2

for all $l=1, \cdots, n, s=0, \cdots, \omega-1$,

$k \in \mathcal{B}_{v}(l+s n) \cap \mathcal{N} \mathcal{V}$.

for $l=1$ to $n$ do

for $s=0$ to $\omega-1$ do

if $\mathcal{B}_{v}(l+s n) \cap \mathcal{N} \mathcal{V} \neq \emptyset$ then

Compute $M_{C_{l+s n} \rightarrow N_{l}}$ as in Fig. 2

for $k \in \mathcal{B}_{v}(l+s n) \cap \mathcal{N} \mathcal{V}$ do

Compute $M_{C_{l+s n} \rightarrow V_{k}}$ as in Fig. 2 end for

else

$\frac{M_{C_{l+s n} \rightarrow N_{l}}(b) \leftarrow}{\exp (\delta)+\exp (-\delta)}\left(c \exp \left((-1)^{1-b} \delta\right)+\right.$

$\left.(1-c) \exp \left((-1)^{b} \delta\right)\right)$, for $b \in\{0,1\}$, where $c$ equals the fixed value of check node $C_{l+s n}$.

end if

end for

end for

if $M_{C_{q} \rightarrow V_{k}}$ converge for all $q=1, \cdots, n \omega$ and

$k \in \mathcal{B}_{v}(q) \cap \mathcal{N} \mathcal{V}$ then

Break

end if

end for

for $k \in \mathcal{N} \mathcal{V}$ do

$p\left(V_{k}=b\right) \leftarrow \prod_{q \in \mathcal{A}_{c}(k)} M_{C_{q} \rightarrow V_{k}}(b)$, for $b \in\{0,1\}$

$p\left(V_{k}=b\right) \leftarrow \frac{p\left(V_{k}=b\right)}{p\left(V_{k}=0\right)+p\left(V_{k}=1\right)}$, for $b \in\{0,1\}$

if $\left|p\left(V_{k}=0\right)-p\left(V_{k}=1\right)\right|>\eta$ then

$\mathcal{N} \mathcal{V} \leftarrow \mathcal{N} \mathcal{V}-\{k\}$

$\hat{b} \leftarrow \operatorname{argmax}_{b \in\{0,1\}} p\left(V_{k}=b\right), V_{k} \leftarrow \hat{b}$

$M_{V_{k} \rightarrow C_{q}}(\hat{b}) \leftarrow 1, M_{V_{k} \rightarrow C_{q}}(1-\hat{b}) \leftarrow 0$,

for all $q \in \mathcal{A}_{c}(k)$

end if

end for

end while

Fig. 3. Message passing algorithm. $\mathcal{N} \mathcal{V}$ denotes the set of indices $k$ of currently non-decimated variable nodes $V_{k}$. The values of parameter $\delta$ in our tests are provided in Section VII. The threshold $\eta$ is set to 0.9 or 0.99 in our experiments. 
denote the distortion region, i.e.,

$$
\begin{aligned}
\mathcal{D} \triangleq & \left\{\left(d_{1}, d_{2}, d_{0}\right): \exists\left(R_{1}, R_{2}\right) \text { such that } R_{1}+R_{2}=R\left(d_{0}\right)\right. \\
& \text { and } \left.\left(R_{1}, R_{2}, d_{1}, d_{2}, d_{0}\right) \in \mathcal{R} \mathcal{D}\right\} .
\end{aligned}
$$

For $d_{0} \geq 0$ let us additionally define

$$
\mathcal{D}\left(d_{0}\right) \triangleq\left\{\left(d_{1}, d_{2}\right):\left(d_{1}, d_{2}, d_{0}\right) \in \mathcal{D}\right\}
$$

Clearly,

$$
\mathcal{D}=\cup_{d_{0}: d_{0} \geq 0}\left\{\left(d_{1}, d_{2}, d_{0}\right):\left(d_{1}, d_{2}\right) \in \mathcal{D}\left(d_{0}\right)\right\} .
$$

Now we proceed to characterize $\mathcal{D}\left(d_{0}\right)$. With no loss of generality, we shall assume $0 \leq d_{0}<\delta$. Since $\mathcal{D}\left(d_{0}\right)$ is convex, it suffices to characterize its supporting lines. Two of the supporting lines are already known, namely $d_{1}=d_{0}$ and $d_{2}=d_{0}$. Therefore, to complete the task, it is enough to solve the following optimization problem, for all $\alpha>0$,

$$
\min _{\left(d_{1}, d_{2}\right) \in \mathcal{D}\left(d_{0}\right)} \alpha d_{1}+d_{2} .
$$

Note that $R\left(d_{0}\right)$ is a strictly convex function of $d_{0}$ for $d_{0} \in$ $[0, \delta)$. Furthermore, if

$$
\begin{gathered}
R\left(d_{0}\right)=R_{1}+R_{2} \geq I\left(X ; X_{0}, X_{1}, X_{2}\right), \quad \text { and } \\
d_{0} \geq \mathbb{E}\left[d\left(X, X_{0}\right)\right],
\end{gathered}
$$

then one must have $X=X_{0} \oplus Z$, where $\oplus$ denotes the modulo-2 addition operation, and $Z \sim \operatorname{Ber}\left(d_{0}\right)$ is independent of $\left(X_{0}, X_{1}, X_{2}\right)$. Therefore, it suffices to specify $p_{X_{1} X_{2} \mid X_{0}}$ in order to determine $p_{X X_{0} X_{1} X_{2}}$ due to the fact that $p_{X X_{0}}$ is completely determined once $d_{0}$ is given and that $X-X_{0}-\left(X_{1}, X_{2}\right)$ form a Markov chain. Now in view of (12), it can be readily shown that

$$
\begin{aligned}
& \min _{\left(d_{1}, d_{2}\right) \in \mathcal{D}\left(d_{0}\right)} \alpha d_{1}+d_{2} \geq \\
& \min _{p_{X_{1} X_{2} \mid X_{0}}: I\left(X_{1} ; X_{2}\right)=0} \alpha \mathbb{E}\left[d\left(X, X_{1}\right)\right]+\mathbb{E}\left[d\left(X, X_{2}\right)\right] .
\end{aligned}
$$

It turns out that the inequality can be replaced by an equality. To see this, for any $p_{X_{1} X_{2} \mid X_{0}}$ such that the induced $X_{1}$ and $X_{2}$ are independent, let

$$
\begin{aligned}
& R_{1}=I\left(X ; X_{1}\right), \\
& R_{2}=I\left(X, X_{1} ; X_{2}\right)+I\left(X ; X_{0} \mid X_{1}, X_{2}\right), \\
& d_{t}=\mathbb{E}\left[d\left(X, X_{t}\right)\right], \quad t=0,1,2 .
\end{aligned}
$$

It can be verified that $\left(R_{1}, R_{2}, d_{1}, d_{2}, d_{0}\right) \in \mathcal{Q}$ and $R_{1}+R_{2}=$ $R\left(d_{0}\right)$. Therefore, (13) is equivalent to

$$
\min _{p_{X_{1} X_{2} \mid X_{0}}: I\left(X_{1} ; X_{2}\right)=0} \alpha \mathbb{E}\left[d\left(X, X_{1}\right)\right]+\mathbb{E}\left[d\left(X, X_{2}\right)\right] .
$$

Solving (14) for all $\alpha>0$ leads to the following result, whose proof is deferred to Appendix B.

Theorem 2. The following relation holds

$$
\begin{gathered}
\mathcal{D}\left(d_{0}\right)=\left\{\left(d_{1}, d_{2}\right):\left(d_{1}+1-2 d_{0}-\delta\right)\left(d_{2}+1-2 d_{0}-\delta\right)\right. \\
\left.\geq\left(1-d_{0}-\delta\right)\left(1-2 d_{0}\right), d_{1} \geq d_{0}, d_{2} \geq d_{0}\right\}
\end{gathered}
$$

Moreover, for each $\alpha>0$, the solution $\left(d_{1}, d_{2}\right)$ to (13) is unique, and a corresponding solution to (14) is as specified in Fig. 4. Additionally, when $\delta<1 / 2$, the solution to (14) is unique, while for $\delta=1 / 2$ and $d_{1} \neq d_{0}, d_{2} \neq d_{0}$, there is only

$$
\begin{aligned}
& p_{X X_{1} X_{2}}(0,1,1)=\frac{d_{0}}{1-2 d_{0}}\left(1-\delta-d_{0}\right), \\
& p_{X X_{1} X_{2}}(1,0,0)=\frac{d_{0}}{1-2 d_{0}}\left(\delta+d_{0}-d_{1}-d_{2}\right), \\
& p_{X X_{1} X_{2}}(1,1,0)=\frac{d_{0}}{1-2 d_{0}}\left(d_{1}-d_{0}\right), \\
& p_{X X_{1} X_{2}}(1,0,1)=\frac{d_{0}}{1-2 d_{0}}\left(d_{2}-d_{0}\right), \\
& p_{X X_{1} X_{2}}(0,1,0)=\frac{1-d_{0}}{1-2 d_{0}}\left(d_{1}-d_{0}\right), \\
& p_{X X_{1} X_{2}}(0,0,1)=\frac{1-d_{0}}{1-2 d_{0}}\left(d_{2}-d_{0}\right), \\
& p_{X X_{1} X_{2}}(0,0,0)=\frac{1-d_{0}}{1-2 d_{0}}\left(\delta+d_{0}-d_{1}-d_{2}\right), \\
& p_{X X_{1} X_{2}}(1,1,1)=\frac{1-d_{0}}{1-2 d_{0}}\left(1-\delta-d_{0}\right), \\
& X_{0}=\psi_{0}\left(X_{1}, X_{2}\right)=\left\{\begin{array}{c}
1, \\
0,
\end{array} \quad \begin{array}{l}
X_{1}=X_{2}=1 \\
\text { otherwise }
\end{array}\right.
\end{aligned}
$$

Fig. 4. Auxiliary random variables achieving the lower boundary of $\mathcal{D}\left(d_{0}\right)$.

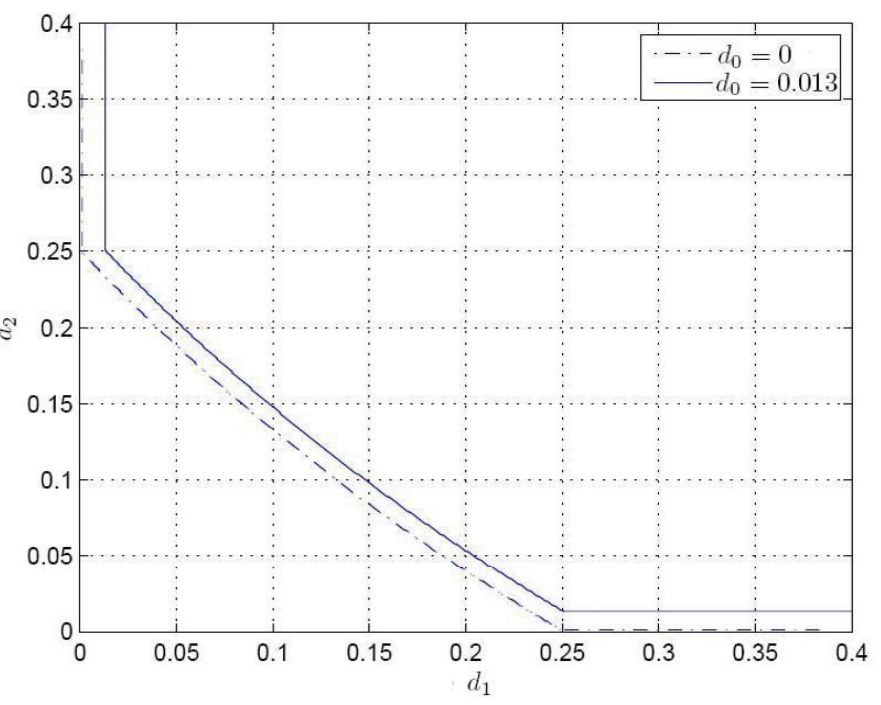

Fig. 5. Distortion region $\mathcal{D}\left(d_{0}\right)$ for a binary source with $p_{X}(0)=1 / 4$, $d_{0}=0$, and $d_{0}=0.013$.

one alternative solution, which is obtained by swapping 0 and 1 in Fig. 4.

Note that for the case of the uniform binary source, part of the conclusion of Theorem 2 can be inferred from [5, Section IV]. Fig. 5 depicts the distortion region $\mathcal{D}\left(d_{0}\right)$ for a binary source with $p_{X}(0)=1 / 4, d_{0}=0$, and $d_{0}=0.013$.

Consider now a distortion pair $\left(d_{1}, d_{2}\right)$ on the lower boundary $^{3}$ of $\mathcal{D}\left(d_{0}\right)$; let $p_{X_{0} X_{1} X_{2} \mid X}$ and $\psi_{0}\left(X_{1}, X_{2}\right)$ be specified by Fig. $4, X_{c}=0$, and $\psi_{t}\left(X_{t}\right)=X_{t}$, for $t=1,2$. Then relations (3) and (4) are satisfied. Further, define the rate region

$$
\begin{gathered}
\mathcal{R}\left(d_{1}, d_{2}, d_{0}\right) \triangleq\left\{\left(R_{1}, R_{2}\right): R_{1}+R_{2}=R\left(d_{0}\right),\right. \text { and } \\
\left.\left(R_{1}, R_{2}, d_{1}, d_{2}, d_{0}\right) \in \mathcal{R} \mathcal{D}\right\} .
\end{gathered}
$$

\footnotetext{
${ }^{3}$ The lower boundary of $\mathcal{D}\left(d_{0}\right)$ is defined as the set of solutions to (13)
} for $\alpha>0$. 
It can be shown by leveraging Theorem 2 that $\mathcal{R}\left(d_{1}, d_{2}, d_{0}\right)$ coincides with the set of rate pairs satisfying $R_{1} \geq I\left(X ; X_{1}\right)$, $R_{2} \geq I\left(X ; X_{2}\right), R_{1}+R_{2}=R\left(d_{0}\right)$. Thus, $\mathcal{R}\left(d_{1}, d_{2}, d_{0}\right)$ coincides with the dominant face $\mathcal{F}\left(p_{X_{c} X_{1} X_{2} \mid X}\right)$ defined in Subsection III-A, and the coding scheme proposed in Section III, with $U_{1}=X_{1}$ and $U_{2}=X_{2}$, can be used to approach its extremities.

\section{No Excess Marginal Rate CASE For the Uniform BINARY SOURCE WITH HAMMING DisTORTION}

The term "no excess marginal rate" refers to the case when $R_{1}=R\left(d_{1}\right)$ and $R_{2}=R\left(d_{2}\right)$. An interesting question in such a case is, given the distortion pair $\left(d_{1}, d_{2}\right)$, what is the minimum distortion $d_{0}$ ? Precisely, let us define

$$
d_{0}\left(d_{1}, d_{2}\right) \triangleq \min \left\{d_{0}:\left(R\left(d_{1}\right), R\left(d_{2}\right), d_{1}, d_{2}, d_{0}\right) \in \mathcal{R} \mathcal{D}\right\} .
$$

Zhang and Berger proposed in [6] an upper bound for $d_{0}\left(d_{1}, d_{2}\right)$ by choosing the auxiliary random variables $X_{c}, X_{1}, X_{2}$ in the definition of the $\mathrm{ZB}$ region such that $X-X_{t}-X_{c}, t=1,2$, and $X_{1}-\left(X, X_{c}\right)-X_{2}$ form Markov chains, $I\left(X ; X_{t}\right)=R\left(d_{t}\right), \mathbb{E}\left[d\left(X, X_{t}\right)\right] \leq d_{t}$, $\psi_{t}\left(X_{t}, X_{c}\right)=X_{t}$, for $t=1,2$, and $\psi_{0}\left(X_{c}, X_{1}, X_{2}\right)=X_{1}$, if $X_{1}=X_{2}$, and $X_{c}$, otherwise.

Further they showed that for the uniform binary source with Hamming distortion the proposed upper bound is strictly better than the upper bound derived from the EGC region, namely than $\min \left(d_{1}, d_{2}\right)$. Notice that the case of interest is when $\min \left(d_{1}, d_{2}\right)>0$. Now let

$$
\begin{aligned}
& \mathbb{P}\left(X_{c}=X \mid X\right)=1-s, \quad \mathbb{P}\left(X_{c} \neq X \mid X\right)=s, \\
& \mathbb{P}\left(X_{t}=X \mid X\right)=1-d_{t}, \quad \mathbb{P}\left(X_{t} \neq X \mid X\right)=d_{t}, \\
& \mathbb{P}\left(X_{c}=X_{t} \mid X\right)=1-s_{t}, \quad \mathbb{P}\left(X_{c} \neq X_{t} \mid X\right)=s_{t}, \quad t=1,2,
\end{aligned}
$$

for some $s$ such that $\max \left(d_{1}, d_{2}\right) \leq s \leq \frac{1}{2}$, and $s_{t}=\frac{s-d_{t}}{1-2 d_{t}}$, $t=1,2$. Then the upper bound is computed in [6] as

$$
U B\left(d_{1}, d_{2}\right)=\inf _{\max \left(d_{1}, d_{2}\right) \leq s \leq \frac{1}{2}} \mathbb{P}\left(X \neq \psi_{0}\left(X_{c}, X_{1}, X_{2}\right)\right) .
$$

We argue that the mapping $\psi_{0}$ given in [6] is not optimal and provide the optimal mapping $\psi_{0}$ which minimizes the central distortion, along with the optimal value $s_{0}$ achieving the infimmum in (15). Let us assume without restricting the generality that $d_{1} \leq d_{2}$. Define $\alpha\left(d_{1}, d_{2}\right) \triangleq \frac{d_{1}\left(1-d_{2}\right)}{d_{1}+d_{2}-2 d_{1} d_{2}}$. Then relations $d_{2} \leq \alpha\left(d_{1}, d_{2}\right) \leq 1 / 2$ hold. In Appendix C we show that the mapping $\psi_{0}$ which minimizes the central distortion is the following

$\psi_{0}\left(x_{c}, x_{1}, x_{2}\right)=\left\{\begin{array}{ccc}x_{1}, & x_{1}=x_{2} \\ 1-x_{c}, & \text { if } x_{1} \neq x_{2}, \quad d_{2} \leq s \leq \alpha\left(d_{1}, d_{2}\right) \\ x_{1}, & \text { if } x_{1} \neq x_{2}, \quad \alpha\left(d_{1}, d_{2}\right)<s \leq 1 / 2\end{array}\right.$

Then the upper bound (15) becomes

$$
\begin{aligned}
& U B\left(d_{1}, d_{2}\right)= \\
& \min _{d_{2} \leq s \leq \alpha\left(d_{1}, d_{2}\right)}\left(1-s-\frac{\left(1-d_{1}\right)\left(1-d_{2}\right)\left(1-s-d_{1}\right)\left(1-s-d_{2}\right)}{(1-s)\left(1-2 d_{1}\right)\left(1-2 d_{2}\right)}\right. \\
& \left.\quad+\frac{d_{1} d_{2}\left(1-s-d_{1}\right)\left(1-s-d_{2}\right)}{s\left(1-2 d_{1}\right)\left(1-2 d_{2}\right)}\right) .
\end{aligned}
$$

Define $\beta\left(d_{1}, d_{2}\right) \triangleq \frac{d_{1}+d_{2}-2 d_{1} d_{2}}{d_{1} d_{2}\left(1-d_{1}\right)\left(1-d_{2}\right)}$. As argued in Appendix
$\mathrm{C}$, the solution $s_{0}$ to (17) is

$$
s_{0}=\frac{1}{2}-\frac{1}{2} \sqrt{1-4 \frac{\sqrt{1+\beta\left(d_{1}, d_{2}\right)}-1}{\beta\left(d_{1}, d_{2}\right)}} .
$$

Thus, $U B\left(d_{1}, d_{2}\right)$ can be recovered by substituting (18) into the expression in (17).

Further, to apply the coding scheme proposed in Section III for the distortion triple $\left(d_{1}, d_{2}, d_{0}=U B\left(d_{1}, d_{2}\right)\right)$, we define $U_{t} \triangleq X_{c} \oplus X_{t}, t=1,2$. It follows easily that $\mathrm{C} 1$ and $\mathrm{C} 3$ are satisfied, while $\mathrm{C} 2$ holds since $X_{t}=\pi_{t}\left(X_{c}, U_{t}\right) \triangleq X_{c} \oplus U_{t}$, $t=1,2$.

\section{Successive Refinement With Hamming DISTORTION}

Successive refinement (SR) can be regarded as a special form of multiple description coding in which the distortion constraint on the second description (i.e., $d_{2}$ ) is not imposed. In this scenario it is common to refer to the first description as the base layer and the second description as the refinement layer. The successive refinement coding rate-distortion region $\mathcal{R} \mathcal{D}_{\mathrm{SR}}$ is given by

$$
\mathcal{R D}_{\mathrm{SR}}=\left\{\left(R_{1}, R_{2}, d_{1}, d_{0}\right):\left(R_{1}, R_{2}, d_{1}, \infty, d_{0}\right) \in \mathcal{R} \mathcal{D}\right\} .
$$

An important case is when $\left(R\left(d_{1}\right), R\left(d_{0}\right)-R\left(d_{1}\right), d_{1}, d_{0}\right) \in$ $\mathcal{R} \mathcal{D}_{\mathrm{SR}}$. The sources for which this relation holds for all admissible $d_{1}>d_{0}$ are called in [19] successively refinable sources. Equitz and Cover showed in [19] that $\left(R\left(d_{1}\right), R\left(d_{0}\right)-\right.$ $\left.R\left(d_{1}\right), d_{1}, d_{0}\right) \in \mathcal{R} \mathcal{D}_{\mathrm{SR}}$ if and only if there are random variables $X_{c}$ and $X_{1}$, jointly distributed with the generic source variable $X$, such that

$$
\begin{aligned}
& R\left(d_{1}\right)=I\left(X ; X_{c}\right), \quad R\left(d_{0}\right)=I\left(X ; X_{c}, X_{1}\right), \\
& \mathbb{E}\left[d\left(X, X_{c}\right)\right] \leq d_{1}, \quad \mathbb{E}\left[d\left(X, X_{1}\right)\right] \leq d_{0},
\end{aligned}
$$

and $X-X_{1}-X_{c}$ form a Markov chain. They further proved, based on the results of [25], that finite alphabet sources with Hamming distortion are successively refinable and that the joint distribution $p_{X X_{c} X_{1}}$ is specified by the relations in Fig. 6 .

$$
\begin{aligned}
& p_{X_{t}}(x)=\frac{\left(p_{X}(x)-\lambda_{t}\right)^{+}}{\sum_{x^{\prime} \in \mathcal{X}}\left(p_{X}\left(x^{\prime}\right)-\lambda_{t}\right)^{+}}, \quad x \in \mathcal{X}, t \in\{c, 1\} \\
& p_{X \mid X_{1}}\left(x \mid x_{1}\right)= \begin{cases}1-d_{0}, & x=x_{1} \in \mathcal{X}_{1}^{+} \\
\lambda_{1}, & x \neq x_{1}, x \in \mathcal{X}_{1}^{+}, x_{1} \in \mathcal{X}_{1}^{+} \\
p_{X}(x) & x \notin \mathcal{X}_{1}^{+}, x_{1} \in \mathcal{X}_{1}^{+}\end{cases} \\
& p_{X_{1} \mid X_{c}}\left(x_{1} \mid x_{c}\right)= \begin{cases}\frac{1-d_{1}-\lambda_{1}}{1-d_{0}-\lambda_{1}}, & x_{1}=x_{c} \in \mathcal{X}_{c}^{+} \\
\frac{\lambda_{c}-\lambda_{1}}{1-d_{0}-\lambda_{1}}, & x_{1} \neq x_{c}, x_{1} \in \mathcal{X}_{c}^{+}, x_{c} \in \mathcal{X}_{c}^{+} \\
\frac{p\left(x_{1}\right)-\lambda_{1}}{1-d_{0}-\lambda_{1}} & x_{1} \in \mathcal{X}_{1}^{+}, x_{1} \notin \mathcal{X}_{c}^{+}, x_{c} \in \mathcal{X}_{c}^{+}\end{cases}
\end{aligned}
$$

where $\lambda_{1} \in\left[0, \lambda_{c}\right], \lambda_{c}$ are determined by

$\sum_{x_{t} \in \mathcal{X}_{t}^{+}} p_{X_{t}}\left(x_{t}\right) p_{X \mid X_{t}}\left(x \mid x_{t}\right)=p_{X}(x), x \in \mathcal{X}, t \in\{c, 1\}$,

$$
\text { and } \mathcal{X}_{t}^{+}=\left\{x \in \mathcal{X}: p_{X}(x)-\lambda_{t}>0\right\}, t \in\{c, 1\}
$$

Fig. 6. Relations defining the joint distribution $p_{X X_{c} X_{1}}$ for SR.

Given $p_{X X_{c} X_{1}}$ as in Fig. 6, the rate-distortion tuple satisfying (19) can be achieved by using a sequential coding scheme adapted from Section III. The scheme consists only 
of encoders 0 and 1, whose outputs represent the base, respectively the refinement layer. To clarify the details of the scheme we only need to specify $U_{1}$. Assume without restricting the generality that $\mathcal{X}=\{0,1, \cdots, \zeta-1\}$ and $\mathcal{X}_{c}^{+}=\{0,1, \cdots, \tau-1\}$, for some integers $\zeta \geq \tau>0$. Then let $U_{1} \triangleq \sigma\left(X_{c}, X_{1}\right)$, where $\sigma\left(X_{c}, X_{1}\right)=X_{1} \oplus_{\tau}\left(\tau-X_{c}\right)$, if $X_{1} \in \mathcal{X}_{c}^{+}$, and $\sigma\left(X_{c}, X_{1}\right)=X_{1}$ otherwise, and $\oplus_{\tau}$ denotes the modulo- $\tau$ addition. It can be easily verified that conditions C1-C3 hold, where $\pi_{1}\left(X_{c}, U_{1}\right)=U_{1} \oplus_{\tau} X_{c}$ if $U_{1} \in \mathcal{X}_{c}^{+}$, and $\pi_{1}\left(X_{c}, U_{1}\right)=U_{1}$ otherwise.

\section{EXPERIMENTAL RESULTS}

We have tested the proposed LDGM-based coding scheme in each of the three cases of the MD problem discussed in the previous section. The degree distributions of the LDGM codes are from the website (http://lthcwww.epfl.ch.research/ldpcopt) or obtained by implementing the algorithm in [26]. We have used damping as in [14], [17] in our message passing algorithm, if the messages do not converge after 30 iterations. The length $n$ of the input sequence is 10,000 . Next we present the experimental results for each case. The values of the empirical distortions are averaged over 100 runs.

\section{A. No Excess Sum-Rate Case for Binary Sources}

We have targeted distortion pairs $\left(d_{1}, d_{2}\right)$ on the lower boundary of $\mathcal{D}\left(d_{0}\right)$ for a uniform binary source (UBS) and for a non-uniform binary source (NBS) with $p_{X}(0)=1 / 4$. In both cases we have considered $d_{0}=0$ and $d_{0}=0.013$. In our tests the sum-rate $R_{1}+R_{2}$ equals $R\left(d_{0}\right)$ in each case. The value of the threshold $\eta$ is 0.9 , while $\delta$ is 1.8 for UBS and 1.6 for NBS. At encoder 1 we set $\gamma(0,0)=$ $\gamma(1,1)=0$, and a) $\gamma(0,1)=1.0, \gamma(1,0) \in[3.2,3.6]$ for UBS; b) $\gamma(0,1) \in[1.0,2.0], \gamma(1,0)=2.8$ for NBS. At encoder 2 we use $\gamma((0,1), 0)=\gamma((1,1), 1)=\gamma((0,0), 0)=$ $\gamma((1,0), 1)=0$ and $\gamma((0,1), 1)=\gamma((1,1), 0)=3.2$, while: a) $\gamma((0,0), 1)=\gamma((1,0), 0) \in[0.0,0.6]$ for UBS; and b) $\gamma((0,0), 1)=\gamma((1,0), 0)=0.0$ for NBS. Tables VII-A and VII-A present the results for UBS, respectively NBS. The second column indicates whether the pair of rates $\left(R_{1}, R_{2}\right)$ is an $E_{1}$ or $E_{2}$ corner point, or it is obtained by timesharing $(T)$ the two corner points. As observed from Tables VII-A and VII-A, the distortions are very close to the theoretical lower bounds.

\section{B. No Excess Marginal Rate Case for the Uniform Binary Source}

We have considered three target distortion triples $\left(d_{1}, d_{2}, d_{0}=U B\left(d_{1}, d_{2}\right)\right): d_{1}=d_{2}=0.1,0.2,0.3$. At encoder 0 , we have set $\gamma\left(x, x_{c}\right)=0$ if $x=x_{c}$, and 0.5 if $x \neq x_{c}$. At encoders 1 and $2, \gamma\left(\left(x, x_{c}\right), u_{1}\right)=\rho\left(x \oplus x_{c}, u_{1}\right)$ and $\gamma\left(\left(x, x_{c}, u_{1}\right), u_{2}\right)=\rho\left(x \oplus x_{c}, u_{2}\right)$, respectively, where $\rho\left(x \oplus x_{c}, u_{t}\right)=0$ if $x \oplus x_{c}=u_{t}$, and 1.2 if $x \oplus x_{c} \neq u_{t}$, $t=1,2$. The value of $\delta$ is 0.5 at encoder 0 and 0.8 at encoders 1 and 2 . The threshold $\eta$ is set to 0.99 . In all the cases $R_{1}=R\left(d_{1}\right)=R_{2}=R\left(d_{2}\right)$. The experimental results, which are summarized in Table III, show very good performance.
TABLE I

TEST RESULTS IN THE NO EXCESS SUM-RATE CASE FOR THE UNIFORM BINARY SOURCE: $\left(d_{1}, d_{2}, d_{0}\right)$ IS A TARGET DISTORTION TRIPLE; $\left(R_{1}, R_{2}\right)$ IS THE PAIR OF RATES USED BY THE CODE; $\hat{d}_{1}, \hat{d}_{2}, \hat{d}_{0}$ ARE THE EMPIRICAL DISTORTIONS.

\begin{tabular}{|c|c|c|c|c|c|}
\hline$\left(R_{1}, R_{2}\right)$ & & $\left(d_{1}, d_{2}, d_{0}\right)$ & $\hat{d}_{1}$ & $\hat{d}_{2}$ & $\hat{d}_{0}$ \\
\hline \hline$(0.500,0.500)$ & $E_{1}$ & $(0.147,0.272,0)$ & 0.154 & 0.279 & 0.008 \\
\hline$(0.500,0.500)$ & $E_{1}$ & $(0.156,0.266,0)$ & 0.156 & 0.269 & 0.008 \\
\hline$(0.383,0.617)$ & $E_{1}$ & $(0.207,0.207,0)$ & 0.209 & 0.212 & 0.007 \\
\hline$(0.617,0.383)$ & $E_{2}$ & $(0.207,0.207,0)$ & 0.212 & 0.209 & 0.007 \\
\hline$(0.500,0.500)$ & $T$ & $(0.207,0.207,0)$ & 0.211 & 0.211 & 0.007 \\
\hline$(0.445,0.555)$ & $E_{1}$ & $(0.174,0.242,0)$ & 0.176 & 0.247 & 0.009 \\
\hline$(0.676,0.324)$ & $E_{2}$ & $(0.174,0.242,0)$ & 0.175 & 0.246 & 0.009 \\
\hline$(0.500,0.500)$ & $T$ & $(0.174,0.242,0)$ & 0.176 & 0.247 & 0.009 \\
\hline$(0.349,0.551)$ & $E_{1}$ & $(0.215,0.215,0.013)$ & 0.219 & 0.216 & 0.021 \\
\hline$(0.551,0.349)$ & $E_{2}$ & $(0.215,0.215,0.013)$ & 0.216 & 0.219 & 0.021 \\
\hline$(0.450,0.450)$ & $T$ & $(0.215,0.215,0.013)$ & 0.218 & 0.218 & 0.021 \\
\hline$(0.422,0.478)$ & $E_{1}$ & $(0.174,0.258,0.013)$ & 0.181 & 0.258 & 0.022 \\
\hline$(0.619,0.281)$ & $E_{2}$ & $(0.174,0.258,0.013)$ & 0.177 & 0.259 & 0.021 \\
\hline$(0.450,0.450)$ & $T$ & $(0.174,0.258,0.013)$ & 0.180 & 0.258 & 0.022 \\
\hline
\end{tabular}

TABLE II

TEST RESULTS IN THE NO EXCESS SUM-RATE CASE FOR A BINARY SOURCE WITH $p_{X}(0)=1 / 4:\left(d_{1}, d_{2}, d_{0}\right)$ IS A TARGET DISTORTION TRIPLE; $\left(R_{1}, R_{2}\right)$ IS THE PAIR OF RATES USED BY THE CODE; $\hat{d}_{1}, \hat{d}_{2}, \hat{d}_{0}$ ARE THE EMPIRICAL DISTORTIONS.

\begin{tabular}{|c|c|c|c|c|c|}
\hline$\left(R_{1}, R_{2}\right)$ & & $\left(d_{1}, d_{2}, d_{0}\right)$ & $\hat{d}_{1}$ & $\hat{d}_{2}$ & $\hat{d}_{0}$ \\
\hline \hline$(0.492,0.319)$ & $E_{1}$ & $(0.116,0.116,0)$ & 0.120 & 0.120 & 0.014 \\
\hline$(0.319,0.492)$ & $E_{2}$ & $(0.116,0.116,0)$ & 0.120 & 0.120 & 0.014 \\
\hline$(0.424,0.387)$ & $T$ & $(0.116,0.116,0)$ & 0.120 & 0.120 & 0.014 \\
\hline$(0.502,0.309)$ & $E_{1}$ & $(0.060,0.176,0)$ & 0.065 & 0.181 & 0.014 \\
\hline$(0.162,0.649)$ & $E_{2}$ & $(0.176,0.060,0)$ & 0.184 & 0.065 & 0.016 \\
\hline$(0.373,0.439)$ & $T$ & $(0.104,0.132,0)$ & 0.110 & 0.137 & 0.015 \\
\hline$(0.291,0.423)$ & $E_{1}$ & $(0.123,0.123,0.013)$ & 0.130 & 0.128 & 0.025 \\
\hline$(0.423,0.291)$ & $E_{2}$ & $(0.123,0.123,0.013)$ & 0.128 & 0.130 & 0.025 \\
\hline$(0.369,0.344)$ & $T$ & $(0.123,0.123,0.013)$ & 0.129 & 0.129 & 0.025 \\
\hline$(0.492,0.222)$ & $E_{1}$ & $(0.060,0.191,0.013)$ & 0.064 & 0.197 & 0.027 \\
\hline$(0.122,0.591)$ & $E_{2}$ & $(0.191,0.060,0.013)$ & 0.198 & 0.066 & 0.027 \\
\hline$(0.377,0.337)$ & $T$ & $(0.101,0.151,0.013)$ & 0.105 & 0.156 & 0.027 \\
\hline
\end{tabular}

\section{Successive Refinement}

We have tested the proposed SR coding scheme for two binary and one ternary sources. The binary sources are 1) uniform binary source; 2) non-uniform binary source with $p_{X}(0)=0.25$. In both cases $\delta=1.6$, and a) at encoder $0, \gamma\left(x, x_{c}\right)=0$ if $x=x_{c}$, and 2.8 if $x \neq x_{c}$; b) at encoder $1, \gamma\left(\left(x, x_{c}\right), u_{1}\right)=0$ if $x \oplus x_{c}=u_{1}$, and 2.8 otherwise. For the uniform ternary source $\delta=1.1$ and a) at encoder 0 , $\gamma\left(x, x_{c}\right)=0$ if $x=x_{c}$, and $\rho$ if $x \neq x_{c}$; b) at encoder 1 , $\gamma\left(\left(x, x_{c}\right), u_{1}\right)=0$ if $x \oplus_{3}\left(3-x_{c}\right)=u_{1}$, and $\rho$ otherwise, where $\rho \in[1.91,2.31]$. The value of $\eta$ is 0.9 or 0.99 . The results of our experiments are presented in Figs. 7, 8 and 9. As seen in these figures, the empirical distortions are very close to the theoretical limits.

\section{CONCLUSION}

This work presents a practical MD coding scheme based on LDGM codes for the Zhang-Berger region. The asymptotical optimality of the scheme is proved for a joint typicality encoder, while a message passing algorithm is used as a practical encoding solution. The application of this scheme 


\begin{tabular}{|c|c|c|c|c|}
\hline$d_{1}=d_{2}$ & $U B\left(d_{1}, d_{2}\right)$ & $\hat{d}_{1}$ & $\hat{d}_{2}$ & $\hat{d}_{0}$ \\
\hline \hline 0.1 & 0.0626 & 0.1159 & 0.1136 & 0.0783 \\
\hline 0.2 & 0.1574 & 0.2161 & 0.2170 & 0.1720 \\
\hline 0.3 & 0.2658 & 0.3173 & 0.3134 & 0.2823 \\
\hline
\end{tabular}

TABLE III

TEST RESULTS IN THE NO EXCESS MARGINAL RATE CASE FOR THE UNIFORM BINARY SOURCE: $d_{1}=d_{2}$ ARE TARGET DISTORTION VALUES FOR THE SIDE DESCRIPTIONS; $U B\left(d_{1}, d_{2}\right)$ IS THE TARGET VALUE FOR THE CENTRAL DISTORTION; $\hat{d}_{1}, \hat{d}_{2}, \hat{d}_{0}$ ARE THE EMPIRICAL DISTORTIONS.

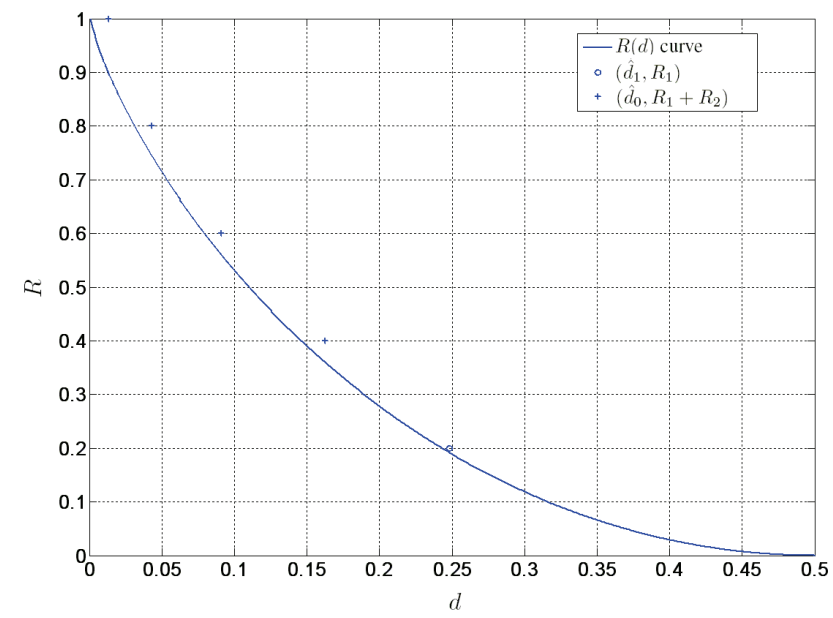

Fig. 7. Simulation results in the SR case for the uniform binary source at $R_{1}=0.2$ and various $R_{2}$ values.

to three cases of the MD problem is discussed in more detail: 1) the no excess sum-rate case for binary sources; 2) the no excess marginal rate case for the uniform binary source; 3) the problem of successive refinement. In all cases, the Hamming distortion is the fidelity criterion. To aid the code design for the first of the aforementioned cases, we provide the analytical expression of the distortion region, which was known previously only for the uniform binary source. Our simulations show very good results, validating the ability of the proposed coding scheme to approach in practice the theoretical rate-distortion limits or the available upper bounds.

\section{APPENDIX A \\ PROOF OF LEMMA 1}

For each $1 \leq l \leq n$, let $\varrho_{l}$ denote the random $m \times \omega$ matrix consisting of columns $l, l+n, l+2 n, \cdots, l+(\omega-1) n$ of the random matrix $\mathbf{G}$, and define $\tilde{Y}^{n}\left(v^{m}\right)=\left(v^{m} \varrho_{l}\right)_{l=1}^{n}$ for every $v^{m} \in \mathbb{F}_{2}^{m}$. Further, for each $y^{n} \in \mathcal{Y}^{n}$ and $\delta>0$ define

$$
\sigma_{n}\left(y^{n}, \mathbf{G}, \delta\right) \triangleq \sum_{v^{m} \in \mathbb{F}_{2}^{m}} \mathbb{I}\left(\left(y^{n}, \tilde{Y}^{n}\left(v^{m}\right)\right)\right) \in \mathcal{T}_{p_{Y, \tilde{Y}}, n}^{\delta,} .
$$

It is easy to verify that if $\left(y^{n}, \tilde{y}^{n}\right) \in \mathcal{T}_{p_{Y, \tilde{Y}}}^{\frac{\delta}{\mid \mathcal{X}}, n}$, then $\left(y^{n}, \phi\left(\tilde{y}^{n}\right)\right) \in \mathcal{T}_{p_{Y, \tilde{Y}}, n}$. Therefore, in order to prove Lemma 1 it is sufficient to show that $\lim _{n \rightarrow \infty} \mathbb{P}\left(\sigma_{n}\left(Y^{n}, \mathbf{G}, \delta\right)>0\right)=1$, for any $\delta>0$.

According to the proof of Theorem 2 in [17] the following holds

$$
\lim _{n \rightarrow \infty} \frac{1}{n} \log _{2} \mathbb{P}\left(\sigma_{n}\left(Y^{n}, \mathbf{G}, \delta\right)>0\right) \geq 0,
$$

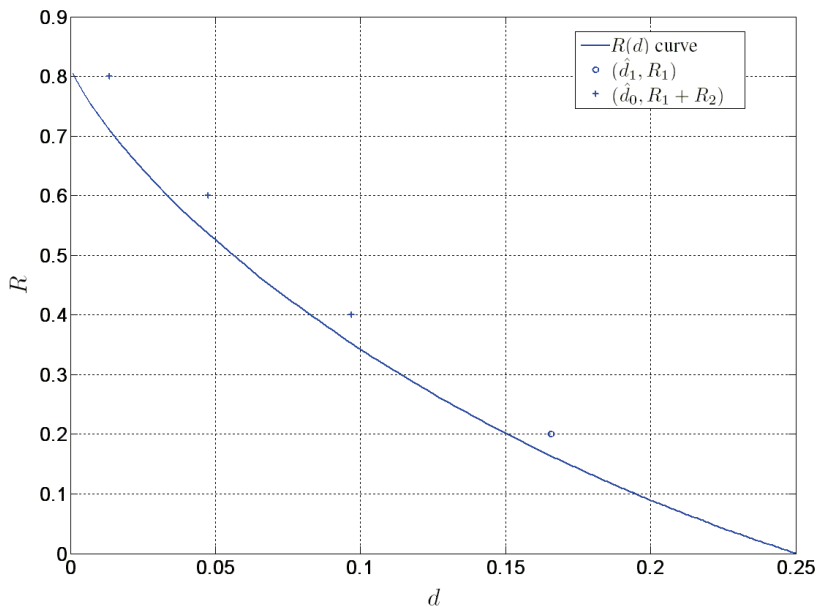

Fig. 8. Simulation results in the SR case for a non-uniform binary source with $p_{X}(0)=0.25$ at $R_{1}=0.2$ and various $R_{2}$ values.

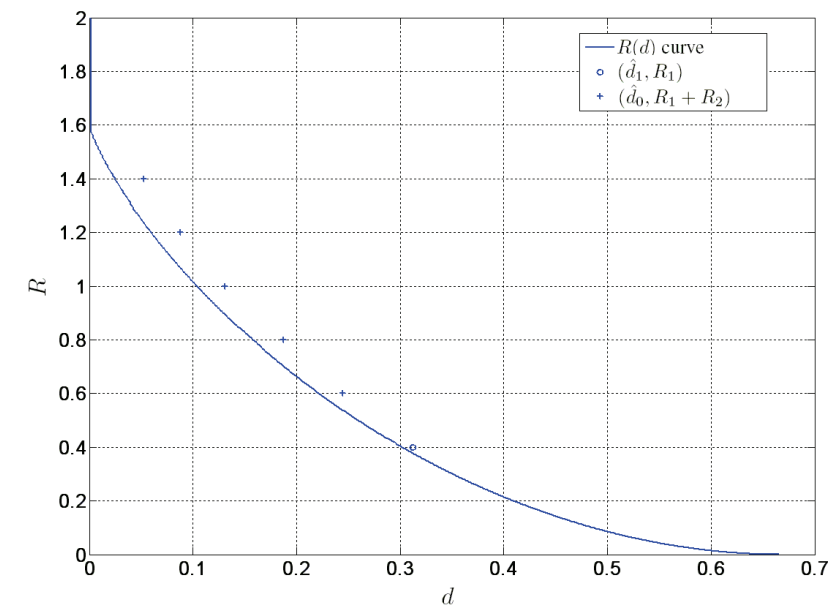

Fig. 9. Simulation results in the SR case for the uniform ternary source at $R_{1}=0.4$ and various $R_{2}$ values.

for any $\delta>0$. We point out that the proof in [17] was carried out assuming that the sequence $Y^{n}$ was i.i.d according to the probability distribution $p_{Y}$. However, a careful examination reveals that only property (7) was needed in the proof ${ }^{4}$.

To complete the proof we will construct a bounded difference martingale sequence. For this, for each $y^{n} \in \mathcal{Y}^{n}$ and matrix $G$ define the mapping

$$
\begin{aligned}
& \rho\left(y^{n}, G\right) \triangleq \\
& \min \left\{\frac{n \alpha}{|\mathcal{Y}||\tilde{\mathcal{Y}}|}:\left(y^{n}, \tilde{y}^{n}\left(v^{m}\right)\right) \in \mathcal{T}_{p_{Y, \tilde{Y}}}^{\alpha, n} \text { for some } v^{m} \in \mathbb{F}_{2}^{m}\right\},
\end{aligned}
$$

where $\tilde{y}^{n}\left(v^{m}\right)$ denotes the realization of $\tilde{Y}^{n}\left(v^{m}\right)$ for the given matrix $G$. Consider now a sequence of random variables $\left\{B_{0, n}, B_{1, n}, \cdots, B_{n, n}\right\}$ defined as follows:

$$
\begin{aligned}
& B_{0, n}=\mathbb{E}\left\{\rho\left(Y^{n}, \mathbf{G}\right)\right\}, \\
& B_{i, n}=\mathbb{E}\left\{\rho\left(Y^{n}, \mathbf{G}\right) \mid Y_{1}, \cdots, Y_{i}, \varrho_{1}, \cdots, \varrho_{i}\right\}, \quad 1 \leq i \leq n .
\end{aligned}
$$

\footnotetext{
${ }^{4}$ In the proof of Theorem 2 of [17] the quantity $I(X ; f(\tilde{X}))$ has to be replaced by $I(X ; \tilde{X})$.
} 
Notice that $B_{n, n}=\rho\left(Y^{n}, \mathbf{G}\right)$. Further, it can be easily shown that the inequality $\left|B_{i+1, n}-B_{i, n}\right| \leq 1$ holds for all $i, 0 \leq i \leq$ $n-1$. Then according to Azuma's inequality [27] it follows that

$$
\mathbb{P}\left\{\left|B_{n, n}-B_{0, n}\right|>n \tau\right\}<2 e^{-\frac{n \tau^{2}}{2}},
$$

for any $n, \tau>0$. Further, using a proof by contradiction one obtains the equality $\lim _{n \rightarrow \infty} \frac{1}{n} B_{0, n}=0^{5}$ based on (20) and (21). Finally, the previous equality corroborated with (21) immediately implies that $\lim _{n \rightarrow \infty} \mathbb{P}\left\{B_{n, n}>n \delta\right\}=0$ for all $\delta>0$, which completes the proof.

\section{APPENDIX B}

\section{PROOF OF THEOREM 2}

Since $\mathcal{D}\left(d_{0}\right)$ is symmetric, it suffices to solve (14) for $\alpha \geq$ 1. Note that

$$
\begin{aligned}
\mathbb{E}\left[d\left(X, X_{t}\right)\right] & =\mathbb{E}\left[d\left(X_{0} \oplus Z, X_{t}\right)\right] \\
& =\mathbb{E}\left[\mathbb{E}\left[d\left(X_{0} \oplus Z, X_{t}\right) \mid Z\right]\right] \\
& =\left(1-d_{0}\right) \mathbb{E}\left[d\left(X_{0}, X_{t}\right)\right]+d_{0}\left(1-\mathbb{E}\left[d\left(X_{0}, X_{t}\right)\right]\right) \\
& =\left(1-2 d_{0}\right) \mathbb{E}\left[d\left(X_{0}, X_{t}\right)\right]+d_{0},
\end{aligned}
$$

for $t=1,2$. Therefore problem (14) is equivalent to

$$
\min _{p_{X_{1} X_{2} \mid X_{0}}: I\left(X_{1} ; X_{2}\right)=0} \alpha \mathbb{E}\left[d\left(X_{0}, X_{1}\right)\right]+\mathbb{E}\left[d\left(X_{0}, X_{2}\right)\right] .
$$

To tackle this minimization problem, we first fix the marginal distribution of $X_{1}$, in other words, let $p_{X_{1}}(0)=\epsilon$, for some $\epsilon, 0<\epsilon<1$, and solve the parameterized problem $P(\epsilon)$, then optimize over all possible values of $\epsilon$. In order to proceed let us adopt the following notation

$$
\begin{array}{ll}
y_{1}=p_{X_{0} X_{1} X_{2}}(0,1,1), & y_{2}=p_{X_{0} X_{1} X_{2}}(1,0,0), \\
y_{3}=p_{X_{0} X_{1} X_{2}}(1,1,0), & y_{4}=p_{X_{0} X_{1} X_{2}}(1,0,1), \\
y_{5}=p_{X_{0} X_{1} X_{2}}(0,1,0), & y_{6}=p_{X_{0} X_{1} X_{2}}(0,0,1), \\
y_{7}=p_{X_{0} X_{1} X_{2}}(0,0,0), & y_{8}=p_{X_{0} X_{1} X_{2}}(1,1,1) .
\end{array}
$$

Then

$$
\begin{aligned}
& d_{1}^{*} \triangleq \mathbb{E}\left[d\left(X_{0}, X_{1}\right)\right]=y_{1}+y_{2}+y_{4}+y_{5}, \\
& d_{2}^{*} \triangleq \mathbb{E}\left[d\left(X_{0}, X_{2}\right)\right]=y_{1}+y_{2}+y_{3}+y_{6} .
\end{aligned}
$$

Let $\mathbf{y}=\left(y_{1}, \cdots, y_{8}\right)$ and $\delta^{*} \triangleq p_{X_{0}}(0)=\frac{\delta-d_{0}}{1-2 d_{0}} \leq 1 / 2$. Now problem (22) becomes

$$
\begin{aligned}
\min _{0<\epsilon<1} \min _{\mathbf{y}} C(\epsilon, \mathbf{y})=(\alpha & +1) y_{1}+(\alpha+1) y_{2} \\
& +y_{3}+\alpha y_{4}+\alpha y_{5}+y_{6}
\end{aligned}
$$

$$
\begin{aligned}
\text { subject to } & \sum_{i=1}^{8} y_{i}=1 \\
& y_{1}+y_{5}+y_{6}+y_{7}=\delta^{*} \\
& y_{2}+y_{4}+y_{6}+y_{7}=\epsilon \\
& y_{2}+y_{7}=\frac{\epsilon}{1-\epsilon}\left(y_{3}+y_{5}\right) \\
& y_{i} \geq 0, \quad 1 \leq i \leq 8 .
\end{aligned}
$$

The second constraint in (25) is obtained from $p_{X_{0}}(0)=\delta^{*}$, while the third follows from $p_{X_{1}}(0)=\epsilon$. The fourth is derived

\footnotetext{
${ }^{5}$ The proof of this relation proceeds as the proof of relation (74) in [16].
}

from $p_{X_{1}}(0) p_{X_{2}}(0)=p_{X_{1} X_{2}}(0,0)$, which holds due to the independence of $X_{1}$ and $X_{2}$. First we solve problem $P(\epsilon)$ for the case when $\epsilon \leq 1 / 2$. From the four equations in (25) one obtains

$$
\begin{aligned}
y_{5}=-y_{1}+y_{2}+y_{4}+\delta^{*}-\epsilon \\
y_{8}=-y_{2}-y_{3}-y_{4}+1-\delta^{*}, \\
y_{6}=\frac{\epsilon}{1-\epsilon} y_{1}-\frac{\epsilon}{1-\epsilon} y_{2}-\frac{\epsilon}{1-\epsilon} y_{3}-\frac{1}{1-\epsilon} y_{4}+\frac{\epsilon\left(1-\delta^{*}\right)}{1-\epsilon}, \\
y_{7}=-\frac{\epsilon}{1-\epsilon} y_{1}+\left(\frac{\epsilon}{1-\epsilon}-1\right) y_{2}+\frac{\epsilon}{1-\epsilon} y_{3}+\frac{\epsilon}{1-\epsilon} y_{4}+ \\
\quad \frac{\epsilon\left(\delta^{*}-\epsilon\right)}{1-\epsilon} .
\end{aligned}
$$

Plugging them into the cost function yields

$$
\begin{aligned}
C(\epsilon, \mathbf{y})= & \alpha\left(\delta^{*}-\epsilon\right)+\frac{\epsilon\left(1-\delta^{*}\right)}{1-\epsilon}+\left(1+\frac{\epsilon}{1-\epsilon}\right) y_{1} \\
& +\left(2 \alpha+1-\frac{\epsilon}{1-\epsilon}\right) y_{2}+\left(1-\frac{\epsilon}{1-\epsilon}\right) y_{3} \\
& +\left(2 \alpha-\frac{1}{1-\epsilon}\right) y_{4} .
\end{aligned}
$$

It can be easily verified that the coefficients of all variables in (27) are non-negative when $\epsilon \leq 1 / 2$, and are strictly positive when $\epsilon<1 / 2$. Thus, $C(\epsilon, \mathbf{y}) \geq g_{1}(\epsilon) \triangleq \alpha\left(\delta^{*}-\epsilon\right)+\frac{\epsilon\left(1-\delta^{*}\right)}{1-\epsilon}$, for any $\mathbf{y}$ satisfying (25). If, additionally, $\epsilon \leq \delta^{*}$, then the vector $\mathbf{y}$ obtained using (26) and $y_{1}=y_{2}=y_{3}=y_{4}=0$ has non-negative components, hence it is a feasible solution achieving the lower bound $g_{1}(\epsilon)$, and therefore, it is a solution to $P(\epsilon)$. Now denote by $C^{*}(\epsilon)$ the value of the cost function of $P(\epsilon)$ at optimality. The conclusion of the above discussion is stated next.

Assertion 1. Let $\alpha \geq 1$. For $0<\epsilon \leq 1 / 2$, one has $C^{*}(\epsilon) \geq$ $g_{1}(\epsilon)$. Additionally, when $0<\epsilon \leq \delta^{*}$, one has $C^{*}(\epsilon)=g_{1}(\epsilon)$ and a solution to $P(\epsilon)$ is $\mathbf{y}(\epsilon)$ defined by

$$
\begin{aligned}
& y_{1}(\epsilon)=y_{2}(\epsilon)=y_{3}(\epsilon)=y_{4}(\epsilon)=0, \\
& y_{5}(\epsilon)=\delta^{*}-\epsilon, \quad y_{6}(\epsilon)=\frac{\epsilon\left(1-\delta^{*}\right)}{1-\epsilon}, \\
& y_{7}(\epsilon)=\frac{\epsilon\left(\delta^{*}-\epsilon\right)}{1-\epsilon}, \quad y_{8}(\epsilon)=1-\delta^{*} .
\end{aligned}
$$

Furthermore, when $0<\epsilon \leq \delta^{*}$ and $\epsilon \neq 1 / 2, \mathbf{y}(\epsilon)$ is the unique solution to $P(\epsilon)$.

The next result can be easily verified.

Assertion 2. For $1 \leq \alpha \leq 1 /\left(1-\delta^{*}\right)$,

$$
\min _{0<\epsilon \leq 1 / 2} g_{1}(\epsilon)=g_{1}\left(\epsilon_{1}\right)
$$

where $\epsilon_{1} \triangleq 1-\sqrt{\left(1-\delta^{*}\right) / \alpha} \in(0,1 / 2)$, and $\epsilon_{1}$ is the unique point of minimum.

Next we will analyze the case when $\epsilon>1 / 2$. By solving the variables $y_{3}, y_{4}, y_{7}, y_{8}$ from the four equalities in (25) and replacing them in the cost function, one obtains

$$
\begin{gathered}
C(\epsilon, \mathbf{y})=\alpha\left(\epsilon-\delta^{*}\right)+\frac{\delta^{*}(1-\epsilon)}{\epsilon}+\left(2 \alpha+1-\frac{1-\epsilon}{\epsilon}\right) y_{1}+ \\
\left(1+\frac{1-\epsilon}{\epsilon}\right) y_{2}+\left(2 \alpha-\frac{1}{\epsilon}\right) y_{5}+\left(1-\frac{1-\epsilon}{\epsilon}\right) y_{6} .
\end{gathered}
$$

Because $1 / 2<\epsilon<1$ and $\alpha \geq 1$, the coefficients of variables 
in (28) are non-negative; therefore, we obtain that $C^{*}(\epsilon) \geq$ $g_{2}(\epsilon) \triangleq \alpha\left(\epsilon-\delta^{*}\right)+\frac{\delta^{*}(1-\epsilon)}{\epsilon}$. Further, the following assertion can be easily verified.

Assertion 3. Let $\alpha \geq 1$ and $1 / 2<\epsilon<1$. Then

$$
C^{*}(\epsilon) \geq \min _{1 / 2<\epsilon<1} g_{2}(\epsilon) \geq \min _{0<\epsilon<1} g_{2}(\epsilon)=g_{2}\left(\epsilon_{2}\right),
$$

where $\epsilon_{2} \triangleq \sqrt{\delta^{*} / \alpha} \in(0,1)$.

Finally, we need one more result, which is stated next.

Assertion 4. For $\delta<1 / 2, g_{1}\left(\epsilon_{1}\right)<g_{2}\left(\epsilon_{2}\right)$ holds, while for $\delta=1 / 2$, one has $g_{1}\left(\epsilon_{1}\right)=g_{2}\left(\epsilon_{2}\right)$.

Proof of Assertion 4. The nontrivial case is when $\delta<1 / 2$. Relation $g_{1}\left(\epsilon_{1}\right)<g_{2}\left(\epsilon_{2}\right)$ is equivalent to $2 \sqrt{\alpha}\left(\sqrt{1-\delta^{*}}-\right.$ $\left.\sqrt{\delta^{*}}\right)<(\alpha+1)\left(\left(1-\delta^{*}\right)-\delta^{*}\right)$. By multiplying both sides of the above inequality with $1 /\left(\sqrt{1-\delta^{*}}-\sqrt{\delta^{*}}\right)$, which is positive for $\delta<1 / 2$, it reduces to $2 \sqrt{\alpha}<(\alpha+1)\left(\sqrt{1-\delta^{*}}+\sqrt{\delta^{*}}\right)$, which holds due to $0<2 \sqrt{\alpha} \leq \alpha+1$ and $1<\sqrt{1-\delta^{*}}+\sqrt{\delta^{*}}$.

Further, by combining Assertions 1-4, we conclude that when $1 \leq \alpha \leq 1 /\left(1-\delta^{*}\right)$ a solution to problem (24) is $\left(\epsilon_{1}, \mathbf{y}\left(\epsilon_{1}\right)\right)$. Additionally, when $\delta<1 / 2$ this solution is unique. This solution yields the following values for $d_{1}, d_{2}$ (via (23))

$$
\begin{aligned}
& d_{1}=d_{0}+\left(1-2 d_{0}\right)\left(\sqrt{1-\delta^{*}}\left(1 / \sqrt{\alpha}-\sqrt{1-\delta^{*}}\right)\right), \\
& d_{2}=d_{0}+\left(1-2 d_{0}\right)\left(\sqrt{1-\delta^{*}}\left(\sqrt{\alpha}-\sqrt{1-\delta^{*}}\right)\right) .
\end{aligned}
$$

Further, eliminating $\alpha$ from the above relations leads to

$$
\begin{gathered}
\left(d_{1}+1-2 d_{0}-\delta\right)\left(d_{2}+1-2 d_{0}-\delta\right) \\
=\left(1-d_{0}-\delta\right)\left(1-2 d_{0}\right) .
\end{gathered}
$$

Notice that for any pair $\left(d_{1}, d_{2}\right)$ with $d_{0} \leq d_{1} \leq d_{2}$ satisfying (30), the value of $\alpha$ for which equations (29) hold is $\alpha=$ $\frac{\left(1-d_{0}-\delta\right)\left(1-2 d_{0}\right)}{\left(d_{1}+1-2 d_{0}-\delta\right)^{2}}$, which clearly satisfies $1 \leq \alpha \leq 1 /\left(1-\delta^{*}\right)$. Thus, we have proved that the set of solutions to problem (13) for all $1 \leq \alpha \leq 1 /\left(1-\delta^{*}\right)$ equals the portion of hyperbola (30) corresponding to $d_{0} \leq d_{1} \leq d_{2}$. The solution pair $\left(d_{1}, d_{2}\right)$ corresponding to $\alpha=1 /\left(1-\delta^{*}\right)$ is $\left(d_{1}, d_{2}\right)=\left(d_{0}, \delta\right)$. Since the value of $d_{1}$ in the solution to (13) is non-increasing as $\alpha$ increases, and cannot go below $d_{0}$, it follows that for any $\alpha>$ $1 /\left(1-\delta^{*}\right)$ the unique solution to problem (13) is $\left(d_{1}, d_{2}\right)=$ $\left(d_{0}, \delta\right)$. Using further the fact that $\mathcal{D}\left(d_{0}\right)$ is symmetric the first conclusion of Theorem 2 follows.

To complete the proof note that, for any pair $\left(d_{1}, d_{2}\right)$ with $d_{1} \leq d_{2}$ satisfying (30), the joint probability distribution $p_{X_{0} X_{1} X_{2}}$ can be recovered from the solution vector $\mathbf{y}\left(\epsilon_{1}\right)$. Further, $p_{X X_{1} X_{2}}$ can be evaluated using the Markov chain $X-X_{0}-\left(X_{1}, X_{2}\right)$, leading to relations in Fig. 4. Additionally, since $\mathcal{D}\left(d_{0}\right)$ is symmetric and relations in Fig. 4 are symmetric in $\left(X_{1}, d_{1}\right)$ and $\left(X_{2}, d_{2}\right)$, one concludes that they also hold for $d_{1}>d_{2}$.

We have already established the uniqueness of the conditional probability $p_{X_{0} X_{1} X_{2} \mid X}$ when $\delta<1 / 2$. For the case $\delta=1 / 2$ the range of interest for $\alpha$ is $1 \leq \alpha \leq 1 /\left(1-\delta^{*}\right)=2$. Then $\epsilon_{2}=\sqrt{\frac{1}{2 \alpha}} \geq 1 / 2$ and by Assertions $1-4$, one has $g_{2}\left(\epsilon_{2}\right)=\min _{0<\epsilon<1} C^{*}(\epsilon)$. Note that when $\epsilon=\epsilon_{2}$ and $\alpha<2$ the coefficients of $y_{1}, y_{2}, y_{5}, y_{6}$ in (28) are positive.
Therefore, in order to achieve the minimum value $g_{2}\left(\epsilon_{2}\right)$ the variables $y_{1}, y_{2}, y_{5}, y_{6}$ must be set to 0 . By letting $\epsilon=\epsilon_{2}$ and $y_{1}=y_{2}=y_{5}=y_{6}=0$ in (25), one obtains the only other solution $\left(\epsilon_{2}, \mathbf{y}\left(\epsilon_{2}\right)\right)$ to problem (24). It can be verified that the random variables $X_{1}^{\prime}$ and $X_{2}^{\prime}$ derived from $\left(\epsilon_{2}, \mathbf{y}\left(\epsilon_{2}\right)\right)$ satisfy $p_{X X_{1}^{\prime} X_{2}^{\prime}}\left(x, x_{1}, x_{2}\right)=p_{X X_{1} X_{2}}\left(x \oplus 1, x_{1} \oplus 1, x_{2} \oplus 1\right)$.

\section{APPENDiX C \\ Proof of Claims in Section V}

Using the fact that $X-X_{t}-X_{c}, t=1,2$, and $X_{1}-$ $\left(X, X_{c}\right)-X_{2}$ form Markov chains, one obtains the equation at the top of next page. Then the mapping $\psi_{0}(\cdot)$ which minimizes the central distortion must satisfy

$$
\begin{aligned}
\psi_{0}\left(x_{c}, x_{1}, x_{2}\right) & =\arg \max _{x=0,1} p_{X X_{c} X_{1} X_{2}}\left(x, x_{c}, x_{1}, x_{2}\right) \\
& =\arg \max _{b=0,1} h(b),
\end{aligned}
$$

where $h(b) \triangleq \frac{p_{X \mid X_{1}}\left(b \mid x_{1}\right) p_{X \mid X_{2}}\left(b \mid x_{2}\right)}{p_{X \mid X_{c}}\left(b \mid x_{c}\right)}$. Notice that $p_{X \mid X_{t}}\left(b \mid x_{t}\right)=p_{X_{t} \mid X}\left(x_{t} \mid b\right)$ and $p_{X \mid X_{c}}\left(b \mid x_{c}\right)=p_{X_{c} \mid X}\left(x_{c} \mid b\right)$. Using the fact that the function $\frac{1-x}{x}$ is decreasing for $x>0$, and that $\frac{1-x}{x} \geq 1$ for $0<x \leq 1 / 2$, combined with $0<d_{1} \leq d_{2} \leq s$, it follows easily that $\frac{h(b)}{h(1-b)} \geq 1$, for the following cases: 1) $x_{1}=x_{2}=x_{c}=b$; 2) $x_{1}=x_{2}=b, x_{c}=1-b$; 3) $x_{1}=b, x_{2}=x_{c}=1-b$.

Consider now the case $x_{1}=x_{c}=b$ and $x_{2}=1-b$. Then $\frac{h(1-b)}{h(b)}=\frac{d_{1}}{1-d_{1}} \frac{1-d_{2}}{d_{2}} \frac{1-s}{s}$. Clearly, $\frac{h(1-b)}{h(b)} \geq 1$ holds if and only if $s \leq \alpha\left(d_{1}, d_{2}\right)$. Notice that $d_{2} \leq \alpha\left(d_{1}, d_{2}\right) \leq 1 / 2$. Based on the above arguments one concludes that (16) is valid.

Now let us analyze the central distortion when $s \leq$ $\alpha\left(d_{1}, d_{2}\right)$. The following relations hold

$$
\begin{gathered}
\mathbb{P}\left(X \neq \psi_{0}\left(X_{c}, X_{1}, X_{2}\right)\right) \\
=\mathbb{P}\left(X_{c}=X\right)-\mathbb{P}\left(X=X_{c}=X_{1}=X_{2}\right) \\
+\mathbb{P}\left(X \neq X_{c}, X_{c}=X_{1}=X_{2}\right) \\
=1-s-\frac{\left(1-d_{1}\right)\left(1-d_{2}\right)\left(1-s-d_{1}\right)\left(1-s-d_{2}\right)}{(1-s)\left(1-2 d_{1}\right)\left(1-2 d_{2}\right)} \\
+\frac{d_{1} d_{2}\left(1-s-d_{1}\right)\left(1-s-d_{2}\right)}{s\left(1-2 d_{1}\right)\left(1-2 d_{2}\right)} .
\end{gathered}
$$

Let $f(s)$ denote the last expression. The function $f(\cdot)$ is strictly convex over the interval $\left(d_{2}, \alpha\left(d_{1}, d_{2}\right)\right)$ and $f\left(d_{2}\right)=$ $f\left(\alpha\left(d_{1}, d_{2}\right)\right)$. Then its unique point of minimum $s_{0} \in$ $\left(d_{2}, \alpha\left(d_{1}, d_{2}\right)\right)$ is the solution to $f^{\prime}(s)=0$. The above equation is equivalent to $\beta\left(d_{1}, d_{2}\right) s^{2}(1-s)^{2}+2 s(1-s)-1=0$, whose only real solution smaller than $1 / 2$ is given by (18).

\section{REFERENCES}

[1] L. Ozarow, "On a source coding problem with two channels and three receivers," Bell Syst. Tech. J., vol. 59, pp. 1909-1921, 1980.

[2] J. K. Wolf, A. D. Wyner, and J. Ziv, "Source coding for multiple descriptions," Bell Syst. Tech. J., vol. 59, pp. 1417-1426, 1980.

[3] A. El Gamal and T. M. Cover, "Achievable rates for multiple descriptions," IEEE Trans. Inf. Theory, vol. IT-28, no. 6, pp. 851-857, Nov. 1982.

[4] R. Ahlswede, "The rate-distortion region for multiple descriptions without excess rate," IEEE Trans. Inf. Theory, vol. IT-31, pp. 721-726, Nov. 1985.

[5] Z. Zhang and T. Berger, "New results in binary multiple descriptions," IEEE Trans. Inf. Theory, vol. IT-33, pp. 502-521, July 1987.

[6] Z. Zhang and T. Berger, "Multiple description source coding with no excess marginal rate," IEEE Trans. Inf. Theory, vol. 41, no. 2, pp. 502521, Mar. 1995. 


$$
p_{X X_{c} X_{1} X_{2}}\left(x, x_{c}, x_{1}, x_{2}\right)=\frac{p_{X_{1}}\left(x_{1}\right) p_{X \mid X_{1}}\left(x \mid x_{1}\right) p_{X_{c} \mid X_{1}}\left(x_{c} \mid x_{1}\right) p_{X_{2}}\left(x_{2}\right) p_{X \mid X_{2}}\left(x \mid x_{2}\right) p_{X_{c} \mid X_{2}}\left(x_{c} \mid x_{2}\right)}{p_{X_{c}}\left(x_{c}\right) p_{X \mid X_{c}}\left(x \mid x_{c}\right)}
$$

[7] J. Wang, J. Chen, L. Zhao, P. Cuff, and H. Permuter, "On the role of the refinement layer in multiple description coding and scalable coding," IEEE Trans. Inf. Theory, vol. 57, pp. 1443-1456, Mar. 2011.

[8] V. A. Vaishampayan, "Design of multiple description scalar quantizers," IEEE Trans. Inf. Theory, vol. 39, pp. 821-834, May 1993.

[9] V. K Goyal and J. Kovacevic, "Generalized multiple description coding with correlating transforms," IEEE Trans. Inf. Theory, vol. 47, pp. 21992224, Sep. 2001.

[10] V. A. Vaishampayan, N. Sloane, and S. Servetto, "Multiple description vector quantization with lattice codebooks: design and analysis," IEEE Trans. Inf. Theory, vol. 47, pp. 1718-1734, July 2001.

[11] S. Dumitrescu and X. Wu, "Optimal two-description scalar quantizer design," Algorithmica, vol. 41, no. 4, pp. 269-287, Feb. 2005.

[12] J. Chen, C. Tian, T. Berger, and S. S. Hemami, "Multiple description quantization via Gram-Schmidt orthogonalization," IEEE Trans. Inf. Theory, vol. 52, pp. 5197-5217, Dec. 2006.

[13] M. Wainwright and E. Maneva, "Lossy source compression using lowdensity generator matrix codes: analysis and algorithms," IEEE Trans. Inf. Theory, vol. 56, no. 3, pp. 1351-1368, Mar. 2010.

[14] T. Filler and J. Friedrich, "Binary quantization using belief propagation with decimation over factor graphs of LDGM codes," in Proc. Allerton Conf. Commun., Control, Comput., Monticello, IL, Sept. 2007.

[15] M. J. Wainwright and E. Martinian, "Low-density graph codes that are optimal for binning and coding with side information," IEEE Trans. Inf. Theory, vol. 55, no. 3, pp. 1061-1079, Mar. 2009.

[16] A. Gupta and S. Verdu, "Nonlinear sparse-graph codes for lossy compression," IEEE Trans. Inf. Theory, vol. 55, no. 5, pp. 1961-1975, May 2009.

[17] Z. Sun, M. Shao, J. Chen, K. M. Wong, and X. Wu, "Achieving the rate-distortion bound with low-density generator matrix codes," IEEE Trans. Commun., vol. 58, pp. 1643-1653, June 2010.

[18] V. N. Koshelev, "Hierarchical coding of discrete sources," Probl. Pered. Inf., vol. 16, pp. 31-49, 1980

[19] W. H. R. Equitz and T. M. Cover, "Successive refinement of information," IEEE Trans. Inf. Theory, vol. 37, pp. 269-275, Mar. 1991.

[20] B. Rimoldi, "Successive refinement of information: characterization of the achievable rates," IEEE Trans. Inf. Theory, vol. 40, pp. 253-259, Jan. 1994.

[21] J. Chen, S. Dumitrescu, Y. Zhang, and J. Wang, "Robust multiresolution coding," IEEE Trans. Commun., vol. 58, no. 11, pp. 3186-3195, Nov. 2010.

[22] J. Chen, Y. Zhang, and S. Dumitrescu, "Gaussian multiple description coding with low-density generator matrix codes," IEEE Trans. Commun., vol. 60, no. 3, pp. 676-687, Mar. 2012.

[23] Y. Zhang, "Practical LDGM-based multiple description coding," Ph.D. thesis, McMaster University, 2011.

[24] T. M. Cover and J. A. Thomas, Elements of Information Theory, 2nd edition. John Wiley \& Sons, 2006.

[25] J. T. Pinkston, "An application of rate-distortion theory to a converse to the coding theorem," IEEE Trans. Inf. Theory, vol. IT-15, pp. 269-275, Jan. 1969 .

[26] S.-Y. Chung, G. D. Forney, T. J. Richardson, and R. Urbanke, "On the design of low-desity parity check codes within $0.0045 \mathrm{~dB}$ of the Shannon limit," IEEE Commun. Lett., vol. 5, pp. 58-60, Feb. 2001.

[27] K. Azuma, "Weighted sums of certain dependent random variables," Tohoku Math. J., vol. 19, pp. 357-367, 1967.

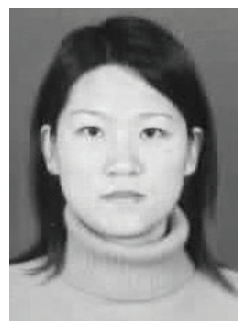

Ying Zhang received the B.Eng degree in Automation from Northeastern University, Shenyang, China in 2004, the M.Eng degree in Pattern Recognition and Intelligence System from Tianjin University, Tianjin, China in 2007 and the Ph.D. degree in Electrical and Computer Engineering from McMaster University, Hamilton, ON, Canada in 2011. Her research interests include information theory and multimedia communications.

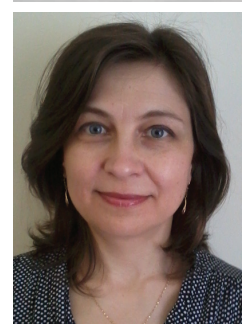

Sorina Dumitrescu (M'05) received the B.A.Sc. and $\mathrm{Ph}$. D. degrees in mathematics from the University of Bucharest, Romania, in 1990 and 1997, respectively.

From 2000 to 2002 she was a Postdoctoral Fellow in the Department of Computer Science at the University of Western Ontario, London, Canada. Since 2002 she has been with the Department of Electrical and Computer Engineering at McMaster University, Hamilton, Canada, where she held Postdoctoral, Research Associate, and Assistant Professor positions, and where she is currently an Associate Professor. Her current research interests include multimedia coding and communications, network-aware data compression, multiple description codes, joint source-channel coding, signal quantization. Her earlier research interests were in formal languages and automata theory. Dr. Dumitrescu has held an NSERC University Faculty Award during 2007-2012.

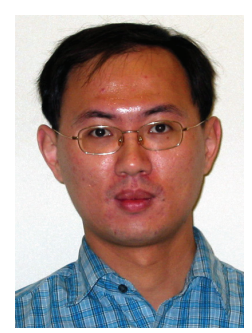

Jun Chen (S'03-M'06) received the B.E. degree with honors in communication engineering from Shanghai Jiao Tong University, Shanghai, China, in 2001 and the M.S. and Ph.D. degrees in electrical and computer engineering from Cornell University, Ithaca, NY, in 2004 and 2006, respectively.

He was a Postdoctoral Research Associate in the Coordinated Science Laboratory at the University of Illinois at Urbana-Champaign, Urbana, IL, from 2005 to 2006, and a Postdoctoral Fellow at the IBM Thomas J. Watson Research Center, Yorktown Heights, NY, from 2006 to 2007. He is currently an Assistant Professor of Electrical and Computer Engineering at McMaster University, Hamilton, ON, Canada. He holds the Barber-Gennum Chair in Information Technology. His research interests include information theory, wireless communications, and signal processing.

He received several awards for his research, including the Josef Raviv Memorial Postdoctoral Fellowship in 2006, the Early Researcher Award from the Province of Ontario in 2010, and the IBM Faculty Award in 2010.

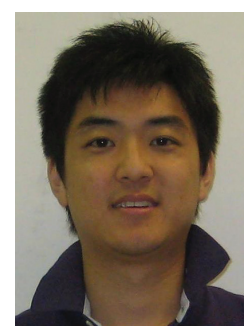

Zhibin Sun received the B.Eng. and M.A.Sc. degrees in electrical engineering from McMaster University, Hamilton, ON, Canada, in 2007 and 2009, respectively. 